\title{
DESCRIPTION OF PERSONNEL PROVISION OF HEALTH CARE SYSTEM OF UKRAINE
}

\section{Slabkiy G. 0.}

\section{INTRODUCTION}

WHO pays much attention to the issues of medical personnel provision of health care system ${ }^{1}$. WHO notes that the effectiveness of health care systems operation depends on their being provided with highly qualified medical personnel ${ }^{2}$. At the same time, the WHO notes the importance of providing with not only doctors, but also with mid-level medical personnel for the effective operation of the system ${ }^{3},{ }_{4}$. It should be noted that during recent years, in the course of medical care, the role of mid-level medical personnel in health care process has significantly increased ${ }^{56}$, . Mid-level medical workers perform not only routine medical services, but also a significant number of medical procedures, which previously were performed exclusively by doctors.

WHO notes that at this stage, almost all countries of the world are experiencing problems with human resources in health care ${ }^{7}$ For their decision WHO approved the Global Strategy for the development of human resources of health care ${ }^{8}$.

\footnotetext{
${ }^{1}$ Junious DL et al. (2004). A study of school nurse job satisfaction. Journal of School Nursing, 20:88-93.

${ }^{2}$ Совместная работа на благо здоровья. Доклад о состоянии здравоохранения в мире, 2006 г.// https://www.who.int/topics/health_workforce/ru/

${ }^{3}$ Razvitie medicinskih kadrov [Medical personnel development]. WHO: website. Retrieved from: http://whodc.mednet.ru/ru/component/attachments/download/20.html (accessed 13 November 2019).

${ }^{4}$ Gupta $\mathrm{N}$ et al. (2003). Uses of population census data for monitoring geographical imbalance in the health workforce: snapshots from three developing countries. International Journal for Equity in Health, 2:11.

${ }^{5}$ Maier, C. B., \& Aiken, L. H. (2016). Task shifting from physicians to nurses in primary care in 39 countries: a cross-country comparative study. European Journal of Public Health, 26(6), 927-934.

${ }^{6}$ Boerma, W.G.W., Kringos, D.S., Wiegers, T.A., Baltag, V., \& Khimion, L. (2010). Evaluation of structure and provision of primary care in Ukraine: a survey-based project in the regions of Kiev and Vinnitsa.

${ }^{7}$ Галієнко Л. І. Сучасні кадрові проблеми охорони здоровя в країнах Європейського регіону ВООЗ. Східноєвропейський журнал громадського здоров'я. 2011. №1 (13). С. 279-281.

${ }^{8}$ Kadry zdravoohraneniya 2030. Globalnaya strategiya dlya razvitiya kadrovyh resursov zdravoohraneniya [Health personnel 2030. Global strategy for health workforce development. WHO: website. Retrieved from: https://www.who.int/hrh/resources/russian_global strategyHRH.pdf (accessed 12 November 2019).
} 
The strengthening of the problem of the provision of health systems by medical personnel in some countries is due to their migration ${ }^{9}$. At the same time, statistically reliable data on the volume of migration of medical workers are absent ${ }^{10}$. Taking into account personnel provision problems which are increasing with regard to labor migration of medical workers WHO adopted The Commonwealth Code of international recruitment of health care personnel, which establishes the guidelines for the international recruitment of medical workers ${ }^{11}$. This document is important also for Ukraine. WHO also pays considerable attention to the issues of attracting and retaining health workers, especially in rural areas ${ }^{12}$.

In order to study the problems associated with personnel resources, WHO developed and recommended for the countries the methodology which is applied at present in Ukraine ${ }^{13}$.

Many scientists in Ukraine are engaged in the problems of human resources in health care: Lekhan V.M. ${ }^{14}$, Slabkiy G.O. ${ }^{15}$, Vezhnovets T.A. ${ }^{16}$, Voronenko Yu.V. ${ }^{17}$, Vasyuk N.O. ${ }^{18}$ et al.

${ }^{9}$ Forcier MB et al. (2004). Impact, regulation and health policy implications of physician migration in OECD countries. Human Resources for Health, 2:12.

${ }^{10}$ Diallo K. Data on the migration of health-care workers: sources, uses, and challenges. Bulletin of the World Health Organization, 2004, 82:559-636.

${ }^{11}$ Commonwealth Code of Practice for the International Recruitment of Health Workers. London, Commonwealth Secretariat, 2003(http://www.thecommonwealth.org/ shared_asp_files/ uploadedfiles/\{7BDD970B-53AE-441D-81DB-

1B64C37E992A\}_CommonwealthCodeofPractice.pdf,accessed 15 April 2008.

12 Rasshirenie dostupa $\mathrm{k}$ rabotnikam zdravoohraneniya $\mathrm{v}$ otdalennyh $\mathrm{i}$ selskih rajonah. Rekomendacii po globalnoj politike. [Increased access to health workers in remote and rural areas. Global policy recommendations]. WHO: website. Retrieved from: http://www.who.int/entity/hrh/ retention/retention_recommendations_ru.pdf (accessed 11 November 2019).

13 Ocenka finansirovaniya, obrazovaniya, upravleniya i politicheskogo konteksta dlya strategicheskogo planirovaniya kadrovyh resursov zdravoohraneniya [Assessment of financing, education, governance and policy context for strategic health workforce planning]. WHO: website. Retrieved from: (http://apps.who.int/gb/ebwha/pdf_files/WHA59-REC1/r/Part2-Resru.pdf) (accessed 14 November 2019).

14 Лехан В. М., Крячкова Л. В. Наукове обгрунтування сучасних підходів до підготовки керівних медичних кадрів. Кадрова політика у сфері охорони здоров'я в умовах загроз національній безпеиі України : матеріали щорічної наук.-практ. конф. за міжнар. участю (Київ, 23 берез. 2017 р.). Київ. С. 86-89.

15 Слабкий Г.О. Кадрова політика. URL : http://www.uiph.kiev.ua/index.asp?p= information\&s $=2$

${ }^{16}$ Вежновець Т. А. До питання визначення критеріївпрофесійного психологічного добору керівників закладів охорони здоров'я. Украйнський журнал з проблем медииини npaui. 2014. №1. С. 28-36.

${ }_{17}^{17}$ Вороненко В., Фещенко І. Кадри забезпечують успіх. Ваме здоров'я. 2011. № 49. URL : http:// www.vz.kiev.ua/?p=3813.

18 Васюк Н. О. Удосконалення кадрового забезпечення державного управління охороною здоров 'я в Україні. Збірник наукових праць Національної академії державного управління при Президентові України. 2015. Вип. 2. С. 34-51 
In their works they indicate the irrational use of medical personnel, low level of their motivation to effective work. The researchers also note the insufficient level of medical skills, mismatch of the ratio of doctors and nurses with the needs, uneven provision of rural residents and residents of cities with medical staff. This situation requires the improvement of personnel policy in health care system of Ukraine.

This is especially important in the conditions of the reform of the healthcare system in the country ${ }^{19}, 20$.

This is especially important in the conditions of the reform of the healthcare system in the country.

In this work we studied and analyzed the issues of provision with doctors and mid-level health workers by the regions of Ukraine for the period of 2014-2018 years.

Statistics of the Ministry of Health of Ukraine and the material of situational analysis "Human resources of the healthcare system in Ukraine" were used in the course of performing this study ${ }^{21}, 22$.

\section{Provision with medicinal personnel in terms of administrative territories}

At first statistics on the provision of the population with doctors in terms of regions of Ukraine was studied and analyzed. The received data are shown in Table 1.

\footnotetext{
${ }^{19}$ Національна стратегія реформування системи охорони здоров'я в Україні на період 2015-2020 років. URL: https://moz.gov.ua/uploads/0/691-strategiya.pdf.

20 Розпорядження Кабінету Міністрів України від 30.11.2016 № 1013-p «Про схвалення Концепції реформи фінансування системи охорони здоров'я». URL: https://zakon.rada.gov.ua/laws/show/1013-2016-p.

21 Державний заклад «Центр медичної статистики Міністерства охорони здоров’я України». URL: http://medstat.gov.ua.

${ }^{22}$ Кадрові ресурси системи охорони здоров'я в Україні. Ситуаційний аналіз / Д. Богдан, А. Бойко, А. Василькова та ін. Проект USAID «Підтримка реформи охорони здоров'я». Київ, 2019. - 133 с.
} 
Table 1

Medical positions in the regions of Ukraine, 2014

\begin{tabular}{|c|c|c|c|c|c|c|c|c|c|c|c|}
\hline Region & 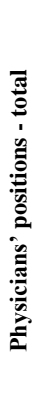 & 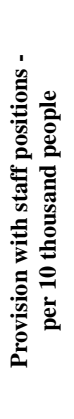 & 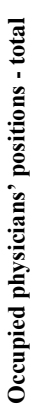 & 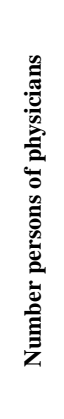 & 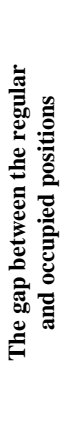 & 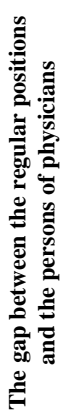 & 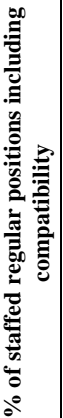 & 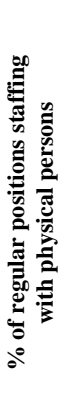 & 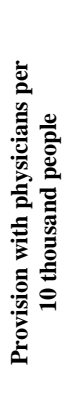 & 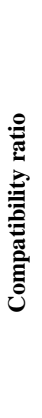 & 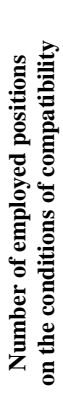 \\
\hline 1 & 2 & 3 & 4 & 5 & 6 & 7 & 8 & 9 & 10 & 11 & 12 \\
\hline Ukraine & 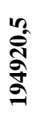 & $\begin{array}{l}\text { की } \\
\text { की }\end{array}$ & 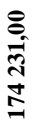 & $\begin{array}{l}\text { है } \\
\text { ì } \\
\text { îे }\end{array}$ & $\begin{array}{l}\text { na. } \\
\hat{0} \\
\text { ते }\end{array}$ & $\begin{array}{l}\frac{n}{n} \\
\frac{n}{4}\end{array}$ & ले & $\begin{array}{l}\text { s. } \\
\text { î }\end{array}$ & $\underset{\text { s }}{F}$ & $\stackrel{g}{=}$ & $\begin{array}{l}\text { s. } \\
\text { î̀ } \\
\text { İ } \\
\text { I }\end{array}$ \\
\hline Vinnitsa & ๙2 & $\overrightarrow{8}$ & ñ & - & $\begin{array}{l}\text { ָ̃ } \\
\stackrel{2}{2}\end{array}$ & $\begin{array}{l}\frac{2}{k} \\
\text { ḋ } \\
\infty\end{array}$ & $\begin{array}{l}F \\
\&\end{array}$ & $\begin{array}{l}2 \\
\\
\text { ฌे }\end{array}$ & $\stackrel{\vec{f}}{\vec{f}}$ & 후 & nू \\
\hline Volyn & 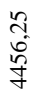 & $\begin{array}{l}+ \\
\infty \\
\sim \\
f\end{array}$ & $\begin{array}{l}n \\
\stackrel{n}{\Xi} \\
\exists\end{array}$ & 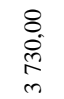 & 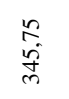 & $\begin{array}{l}\text { â } \\
\text { है } \\
\text { n. }\end{array}$ & $\begin{array}{l}\text { त̃ } \\
\text { }\end{array}$ & $\hat{\infty}$ & $\begin{array}{l}\infty \\
\infty \\
\ddot{m}\end{array}$ & $=$ & $\begin{array}{l}n \\
\tilde{o} \\
\infty \\
m\end{array}$ \\
\hline Dnipropetrovsk & $\begin{array}{l}8 \\
0 \\
0 \\
\text { ర } \\
0\end{array}$ & $\begin{array}{l}\stackrel{2}{\hat{n}} \\
\text { in }\end{array}$ & 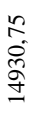 & $\begin{array}{l}8 \\
\dot{\infty} \\
\infty \\
\infty \\
\simeq \\
\simeq\end{array}$ & 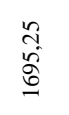 & $\begin{array}{l}8 \\
\text { లi } \\
\infty \\
\infty \\
\text { m }\end{array}$ & $\begin{array}{l}\infty \\
\infty \\
\infty\end{array}$ & $\frac{m}{\approx}$ & ले & $\stackrel{0}{=}$ & $\begin{array}{l}\stackrel{2}{a} \\
\frac{a}{n}\end{array}$ \\
\hline Donetsk & $\begin{array}{l}8 \\
8 \\
\delta \\
\infty \\
\infty\end{array}$ & $\begin{array}{l}\text { o. } \\
\infty \\
\infty\end{array}$ & 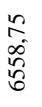 & $\begin{array}{l}8 \\
8 \\
i \\
0 \\
0 \\
i n\end{array}$ & $\begin{array}{l}\stackrel{2}{n} \\
\frac{f}{ \pm}\end{array}$ & $\begin{array}{l}\frac{8}{i} \\
\text { ते } \\
\text { त }\end{array}$ & $\frac{\sigma}{\infty}$ & $\begin{array}{l}\sqrt{n} \\
\text { ภิ }\end{array}$ & $\begin{array}{l}\infty \\
=\end{array}$ & సे & $\begin{array}{l}\frac{n}{a} \\
\text { ma } \\
\dot{g}\end{array}$ \\
\hline Zhytomyr & $\frac{n}{n}$ & $\begin{array}{l}\stackrel{g}{+} \\
\mathfrak{f}\end{array}$ & $\begin{array}{l}8 \\
\infty \\
\infty \\
0 \\
i n\end{array}$ & 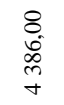 & $\begin{array}{l}n \\
n \\
\infty \\
n \\
n\end{array}$ & $\begin{array}{l}\text { n. } \\
\text { m. } \\
m\end{array}$ & $\begin{array}{l}\dot{8} \\
\dot{8}\end{array}$ & 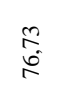 & से & $\stackrel{\infty}{=}$ & $\begin{array}{l}8 \\
\substack{\text { in } \\
\infty \\
\infty}\end{array}$ \\
\hline
\end{tabular}


Continuation Table 1

\begin{tabular}{|c|c|c|c|c|c|c|c|c|c|c|c|}
\hline 1 & 2 & 3 & 4 & 5 & 6 & 7 & 8 & 9 & 10 & 11 & 12 \\
\hline Transcarpathian & $\begin{array}{l}8 \\
o \\
o \\
i n \\
+\end{array}$ & $\begin{array}{l}\text { J } \\
\text { s. }\end{array}$ & 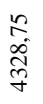 & $\begin{array}{l}8 \\
8 \\
b \\
b \\
+\end{array}$ & $\begin{array}{l}\text { ते } \\
\text { हैं } \\
\text { ते }\end{array}$ & $\begin{array}{l}8 \\
\substack{0 \\
1 \\
1}\end{array}$ & $\begin{array}{l}n \\
\text { ñ } \\
\text { q }\end{array}$ & $\begin{array}{l}8 \\
8 \\
\end{array}$ & $\begin{array}{l}\text { d. } \\
\text { in }\end{array}$ & $\tilde{\sigma}_{0}$ & 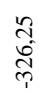 \\
\hline Zaporizhia & $\begin{array}{l}n \\
\text { nn } \\
\infty \\
a\end{array}$ & $\begin{array}{l}\bar{b} \\
\text { in } \\
\text { in }\end{array}$ & 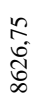 & 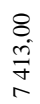 & $\begin{array}{l}\stackrel{n}{2} \\
\stackrel{\infty}{\infty} \\
\stackrel{\infty}{=}\end{array}$ & \begin{tabular}{l} 
ñ \\
aे \\
\multirow{d}{*}{}
\end{tabular} & $\begin{array}{l}\infty \\
\dot{\infty} \\
\dot{\infty} \\
\dot{\infty}\end{array}$ & $\begin{array}{l}\sqrt{n} \\
\text { s. } \\
\end{array}$ & $\begin{array}{l}\underset{\text { d }}{f} \\
\text { for }\end{array}$ & $\stackrel{0}{=}$ & $\begin{array}{l}\frac{n}{2} \\
\stackrel{m}{\Omega}\end{array}$ \\
\hline $\begin{array}{l}\text { Ivano- } \\
\text { Frankivsk }\end{array}$ & à & $\begin{array}{l}\infty \\
\text { î } \\
\text { in }\end{array}$ & 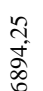 & $\begin{array}{l}8 \\
8 \\
0 \\
0\end{array}$ & 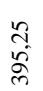 & $\begin{array}{l}n \\
\infty \\
\infty \\
c\end{array}$ & 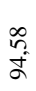 & है & $\begin{array}{l}\text { B. } \\
\text { in }\end{array}$ & oे & 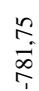 \\
\hline Kyiv & $\begin{array}{l}8 \\
8 \\
\infty \\
\infty \\
\infty\end{array}$ & $\begin{array}{l}\infty \\
\infty \\
\sigma \\
\sigma\end{array}$ & 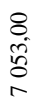 & $\begin{array}{l}\frac{8}{1} \\
\text { ते } \\
\text { D. }\end{array}$ & $\begin{array}{l}8 \\
8 \\
\text { fid } \\
\end{array}$ & 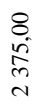 & $\begin{array}{l}n \\
\text { in } \\
\infty\end{array}$ & 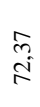 & $\overrightarrow{0}$ & $\cong$ & $\begin{array}{l}8 \\
\text { - } \\
\infty \\
\infty\end{array}$ \\
\hline Kirovohrad & $\begin{array}{l}n \\
\text { o } \\
o \\
b \\
b\end{array}$ & $\begin{array}{l}\stackrel{8}{0} \\
\dot{6}\end{array}$ & $\begin{array}{l}\stackrel{n}{\circ} \\
\stackrel{n}{n} \\
\text { s. }\end{array}$ & $\frac{8}{8}$ & $\begin{array}{l}\text { 足 } \\
\text { s. } \\
\text { nn }\end{array}$ & 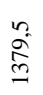 & $\begin{array}{l}\infty \\
\infty \\
\infty \\
\infty \\
\infty\end{array}$ & $\begin{array}{l}50 \\
8 \\
0\end{array}$ & त̂. & $\stackrel{\text { In }}{=}$ & $\begin{array}{l}\bumpeq \\
\sim \\
\infty \\
\infty\end{array}$ \\
\hline Luhansk & $\underset{\substack{n \\
\stackrel{n}{\infty}}}{\stackrel{n}{\infty}}$ & $\begin{array}{l}\delta \\
=\end{array}$ & $\begin{array}{l}8 \\
\stackrel{8}{+} \\
\stackrel{+}{d} \\
\sim\end{array}$ & 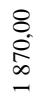 & 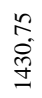 & $\begin{array}{l}n \\
\stackrel{8}{\circ} \\
\stackrel{0}{0}\end{array}$ & $\begin{array}{l}8 \\
\text { ర }\end{array}$ & $\begin{array}{l}\overrightarrow{2} \\
\frac{\sigma}{2}\end{array}$ & $\begin{array}{l}\underset{\infty}{+} \\
\text {. }\end{array}$ & $\stackrel{2}{ }$ & $\begin{array}{l}8 \\
\stackrel{8}{+} \\
\end{array}$ \\
\hline Lviv & $\begin{array}{l}n \\
\tilde{d} \\
\text { d }\end{array}$ & 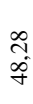 & $\begin{array}{l}\text { a } \\
\text { o } \\
0 \\
\Xi\end{array}$ & 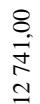 & $\begin{array}{l}\text { in } \\
\text { d } \\
\text { dn }\end{array}$ & $\begin{array}{l}n \\
\infty \\
\infty \\
i\end{array}$ & \begin{tabular}{l}
$\stackrel{0}{2}$ \\
\multirow{8}{\circ}{}
\end{tabular} & $\begin{array}{l}8 \\
8 \\
8\end{array}$ & $\begin{array}{l}\text { in } \\
\text { in }\end{array}$ & $\delta$ & \begin{tabular}{l} 
a \\
$\hat{s}$ \\
\multirow{a}{a}{}
\end{tabular} \\
\hline Mykolaiv & $\begin{array}{l}\text { त̂ } \\
\text { ه్ } \\
\text { ơ }\end{array}$ & $\begin{array}{l}J \\
\text { ले } \\
\text { f }\end{array}$ & 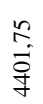 & $\begin{array}{l}8 \\
\text { in } \\
n \\
m \\
m\end{array}$ & $\stackrel{n}{ \pm}$ & $\begin{array}{l}\text { İ } \\
\stackrel{\vec{J}}{\mathcal{I}}\end{array}$ & $\begin{array}{c}m \\
\dot{m}\end{array}$ & $\stackrel{n}{=}$ & ㄱ. & $\stackrel{\text { II }}{=}$ & 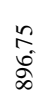 \\
\hline Odesa & $\frac{n}{\vec{I}}$ & $\frac{ \pm}{g}$ & 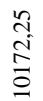 & $\begin{array}{l}8 \\
8 \\
0 \\
0 \\
a\end{array}$ & 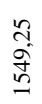 & مَ & $\stackrel{\substack{\infty \\
\infty}}{\widehat{\infty}}$ & $\overbrace{\infty}^{\infty}$ & 哭 & 寓 & $\begin{array}{l}\text { an } \\
\text { f̊ }\end{array}$ \\
\hline Poltava & 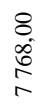 & ते & $\begin{array}{l}8 \\
8 \\
\frac{1}{6} \\
6\end{array}$ & 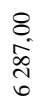 & 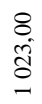 & $\frac{8}{\dot{\alpha}}$ & $\begin{array}{l}\infty \\
\infty \\
\infty\end{array}$ & $\begin{array}{l}\hat{\alpha} \\
\dot{\infty}\end{array}$ & $\begin{array}{l}0 \\
\dot{\gamma} \\
\dot{\gamma}\end{array}$ & s. & $\begin{array}{l}8 \\
\infty \\
\infty \\
q\end{array}$ \\
\hline
\end{tabular}


Continuation Table 1

\begin{tabular}{|c|c|c|c|c|c|c|c|c|c|c|c|}
\hline 1 & 2 & 3 & 4 & $\begin{array}{l}5 \\
\end{array}$ & 6 & 7 & 8 & 9 & 10 & 11 & 12 \\
\hline Rivne & $\begin{array}{l}8 \\
\text { s. } \\
\text { n } \\
\text { in }\end{array}$ & $\begin{array}{l}\bar{m} \\
\vdots \\
q\end{array}$ & $\begin{array}{l}n \\
\infty \\
o \\
\sigma \\
\sigma\end{array}$ & 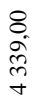 & $\begin{array}{l}\frac{n}{5} \\
i f\end{array}$ & $\begin{array}{l}8 \\
\stackrel{\therefore}{a}\end{array}$ & $\frac{m}{\sigma}$ & $\begin{array}{l}n \\
\text { n. } \\
\infty\end{array}$ & $\underset{\dot{m}}{\stackrel{+}{m}}$ & $\exists$ & $\begin{array}{l}n \\
\text { à } \\
\end{array}$ \\
\hline Sumy & $\begin{array}{l}8 \\
\text { i } \\
\text { in }\end{array}$ & $\begin{array}{l}3 \\
\text { of }\end{array}$ & 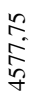 & $\begin{array}{l}8 \\
\text { i } \\
\text { } \\
\text { ma }\end{array}$ & $\begin{array}{l}7 \\
\text { a } \\
\text { f } \\
0\end{array}$ & $\begin{array}{l}8 \\
\stackrel{8}{+} \\
\text { I }\end{array}$ & $\begin{array}{c}= \\
\substack{\infty \\
\infty}\end{array}$ & 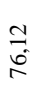 & $\begin{array}{l}\text { ते } \\
\text { ह̂ }\end{array}$ & $\stackrel{0}{=}$ & $\begin{array}{l}n \\
\text { ch } \\
\text { âे }\end{array}$ \\
\hline Ternopil & $\begin{array}{l}\stackrel{n}{2} \\
\text { mp } \\
\stackrel{f}{n}\end{array}$ & $\begin{array}{l}\text { do } \\
\text { in }\end{array}$ & $\begin{array}{l}n \\
\infty \\
\infty \\
n^{2}\end{array}$ & $\begin{array}{l}8 \\
\text { s. } \\
\text { d } \\
\text { in } \\
\text { n. }\end{array}$ & $\begin{array}{l}\text { ñ } \\
\text { D. } \\
m\end{array}$ & $\begin{array}{l}n \\
\infty \\
\infty \\
\infty \\
\infty\end{array}$ & $\begin{array}{l}n \\
n \\
n\end{array}$ & 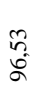 & $=$ & ô & $\begin{array}{l}n \\
\frac{n}{1} \\
\frac{1}{1}\end{array}$ \\
\hline Kharkiv & $\begin{array}{l}\text { a } \\
\text { o } \\
\text { o } \\
\text { ơ }\end{array}$ & $\begin{array}{l}8 \\
\dot{H} \\
\text { in }\end{array}$ & 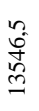 & 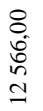 & $\begin{array}{l}n \\
\infty \\
\infty \\
\stackrel{0}{\simeq}\end{array}$ & $\begin{array}{l}\text { ते } \\
\text { ลे } \\
\text { ते }\end{array}$ & 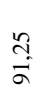 & \begin{tabular}{l}
$n$ \\
\multirow{\infty}{\infty}{} \\
+
\end{tabular} & $\begin{array}{l}\text { त̂ } \\
\text { of }\end{array}$ & $\stackrel{\infty}{\circ}$ & 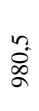 \\
\hline Kherson & 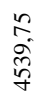 & $\begin{array}{l}\text { in } \\
\text { fi }\end{array}$ & \begin{tabular}{l} 
ra \\
\multirow{2}{*}{$+\frac{1}{q}$}
\end{tabular} & $\begin{array}{l}8 \\
\text { i } \\
\text { d } \\
\text { nat }\end{array}$ & \begin{tabular}{l}
$n$ \\
\multirow{\sigma}{\sigma}{} \\
$\sigma$
\end{tabular} & 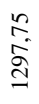 & $\frac{n}{\infty}$ & $\underset{F}{F}$ & 苞 & 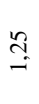 & 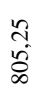 \\
\hline Khmelnitsky & $\begin{array}{l}n \\
\stackrel{2}{0} \\
\hat{\sigma} \\
i n\end{array}$ & $\begin{array}{l}\bar{b} \\
\dot{f}\end{array}$ & 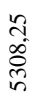 & $\begin{array}{l}8 \\
+ \\
+ \\
0 \\
+\end{array}$ & $\begin{array}{l}n \\
\text { no } \\
o\end{array}$ & $\begin{array}{l}n \\
2 \\
\infty \\
\infty\end{array}$ & $\begin{array}{l}\hat{b} \\
\sigma\end{array}$ & $\begin{array}{l}\vec{n} \\
+ \\
+\end{array}$ & ले & $\stackrel{\infty}{\circ}$ & \begin{tabular}{l} 
वे \\
\multirow{f}{f}{}
\end{tabular} \\
\hline Cherkasy & 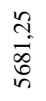 & $\begin{array}{l}\sqrt{2} \\
\text { f }\end{array}$ & $\begin{array}{l}n \\
\text { s. } \\
\approx \\
n\end{array}$ & 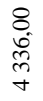 & $\begin{array}{l}n \\
\text { ?. } \\
+ \\
+\end{array}$ & $\begin{array}{l}\text { a } \\
\text { w } \\
\text { s. } \\
\end{array}$ & $\begin{array}{l}f \\
\dot{\sigma}\end{array}$ & 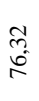 & $\begin{array}{l}\underset{f}{+} \\
\dot{f}\end{array}$ & $\stackrel{-1}{=}$ & $\begin{array}{l}n \\
\hat{0} \\
0 \\
0\end{array}$ \\
\hline Chernivtsi & $\begin{array}{l}8 \\
\circ \\
\infty \\
\infty \\
+\end{array}$ & 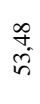 & $\begin{array}{l}\text { 年 } \\
\text { f }\end{array}$ & $\begin{array}{l}\text { o } \\
\text { ij } \\
\text { in } \\
\text { in }\end{array}$ & $\begin{array}{l}n \\
n \\
n \\
n\end{array}$ & 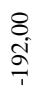 & $\begin{array}{l}\infty \\
m_{0}^{2} \\
\sigma^{2}\end{array}$ & $\begin{array}{l}8 \\
8 \\
8\end{array}$ & $\begin{array}{l}\text { 占 } \\
\text { in }\end{array}$ & مे & in \\
\hline Chernihiv & $\begin{array}{l}n \\
n \\
\infty \\
0 \\
0 \\
n\end{array}$ & $\begin{array}{l}\tilde{N} \\
\stackrel{\delta}{+\infty}\end{array}$ & 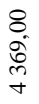 & $\begin{array}{l}8 \\
+\dot{d}^{\circ} \\
\text { m }\end{array}$ & $\begin{array}{l}\frac{2}{0} \\
\hat{\delta}\end{array}$ & 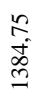 & $\begin{array}{l} \pm \\
0 \\
0 \\
0\end{array}$ & 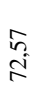 & $\begin{array}{l}\vec{j} \\
\dot{f}\end{array}$ & $\stackrel{\cong}{\rightleftarrows}$ & $\begin{array}{l}8 \\
i \\
i\end{array}$ \\
\hline Kyiv city & 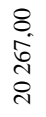 & $\stackrel{2}{i}$ & $\begin{array}{l}\stackrel{n}{a} \\
\text { n. } \\
\stackrel{\infty}{\infty}\end{array}$ & $\begin{array}{l}8 \\
8 \\
0 \\
0 \\
0\end{array}$ & 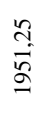 & $\begin{array}{l}8 \\
: \\
5 \\
0\end{array}$ & $\begin{array}{c}\hat{m} \\
\stackrel{2}{2}\end{array}$ & \begin{tabular}{l}
$\infty$ \\
$\infty$ \\
0 \\
\multirow{2}{R}{}
\end{tabular} & $\begin{array}{l}\infty \\
\text { s. } \\
\mathbf{b}^{\circ}\end{array}$ & $\stackrel{\cong}{=}$ & $\begin{array}{l}\text { a } \\
\text { â } \\
\text { d }\end{array}$ \\
\hline
\end{tabular}


Table 2

Medical positions in the regions of Ukraine, 2018

\begin{tabular}{|c|c|c|c|c|c|c|c|c|c|c|c|}
\hline Region & 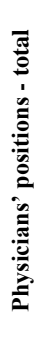 & 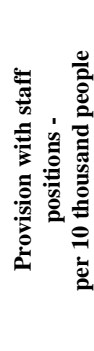 & 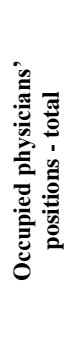 & 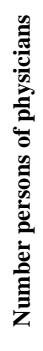 & 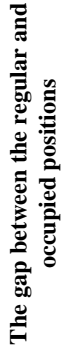 & 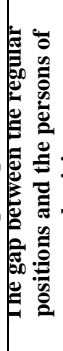 & 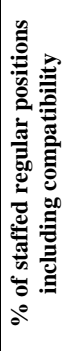 & 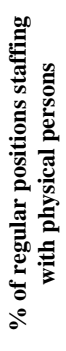 & 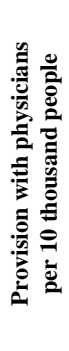 & 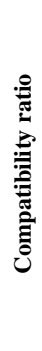 & 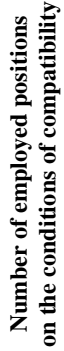 \\
\hline 1 & 2 & 3 & 4 & 5 & 6 & 7 & 8 & 9 & 10 & 11 & 12 \\
\hline Ukraine & 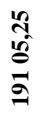 & $\begin{array}{l}F \\
i f\end{array}$ & 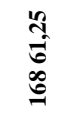 & 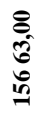 & 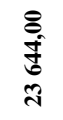 & 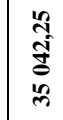 & $\underset{\infty}{\infty}$ & $\frac{\pi}{\infty}$ & m. & $\stackrel{s}{=}$ & $\begin{array}{l}\text { तू } \\
\text { ले } \\
\text { ले }\end{array}$ \\
\hline Vinnitsa & 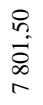 & $\begin{array}{l}\tilde{1} \\
\text { sं }\end{array}$ & $\frac{8}{\grave{~}}$ & $\begin{array}{l}8 \\
\vdots \\
0 \\
1\end{array}$ & $\begin{array}{l}\stackrel{0}{n} \\
\infty \\
\infty \\
\infty\end{array}$ & $\begin{array}{l}\text { ஜn } \\
\text { o̊ } \\
8\end{array}$ & $\begin{array}{l}\vec{N} \\
\infty \\
\infty\end{array}$ & $\frac{\hat{\sigma}}{a}$ & $\underset{⿱ 丶 万}{\stackrel{+}{f}}$ & $\hat{\sigma}$ & $\begin{array}{l}8 \\
+ \\
+ \\
1\end{array}$ \\
\hline Volyn & $\begin{array}{l}8 \\
\infty \\
\infty \\
n \\
+\end{array}$ & $\begin{array}{l}n \\
\infty \\
\tilde{f}\end{array}$ & $\begin{array}{l}n \\
\stackrel{n}{2} \\
\stackrel{2}{n}\end{array}$ & $\begin{array}{l}8 \\
\frac{8}{1} \\
m\end{array}$ & 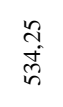 & $\frac{8}{\bar{\infty}}$ & $\begin{array}{l}\text { त) } \\
\infty \\
\infty\end{array}$ & $\begin{array}{l}\text { oे } \\
\text { i }\end{array}$ & $\begin{array}{l}8 \\
\text { s. } \\
\text { D. }\end{array}$ & $\stackrel{5}{0}$ & $\begin{array}{l}\frac{n}{6} \\
\frac{6}{2}\end{array}$ \\
\hline Dnipropetrovsk & 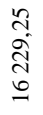 & $\begin{array}{l}\hat{\sigma} \\
\therefore \\
\text { in }\end{array}$ & $\begin{array}{l}8 \\
8 \\
8 \\
\pm\end{array}$ & $\begin{array}{l}8 \\
i \\
\Varangle \\
\simeq\end{array}$ & 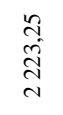 & $\begin{array}{l}\stackrel{2}{2} \\
\frac{1}{\infty} \\
m\end{array}$ & $\begin{array}{l}\text { లి } \\
\text { రి. } \\
\infty\end{array}$ & $\stackrel{\infty}{\stackrel{\infty}{\circ}}$ & $\begin{array}{l}n \\
\stackrel{n}{\infty} \\
\infty\end{array}$ & $\stackrel{m}{=}$ & 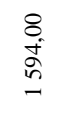 \\
\hline Donetsk & $\begin{array}{l}\stackrel{n}{n} \\
\tilde{n} \\
\infty \\
\infty\end{array}$ & $\begin{array}{l}\text { m. } \\
\text { fr }\end{array}$ & \begin{tabular}{l}
$n$ \\
\multirow{6}{0}{} \\
\multirow{1}{*}{} \\
0
\end{tabular} & $\begin{array}{l}8 \\
\text { i } \\
i n\end{array}$ & $\begin{array}{l}\stackrel{7}{=} \\
= \\
-\end{array}$ & $\begin{array}{l}0 \\
\infty \\
\infty \\
\infty \\
\text { N }\end{array}$ & $\frac{N}{\hat{\infty}}$ & $\frac{0}{8}$ & 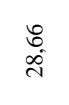 & กิ & $\begin{array}{l}\text { ป̃ } \\
\text { ป } \\
\text { }\end{array}$ \\
\hline 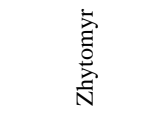 & 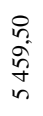 & $\begin{array}{l}\bar{\nabla} \\
\dot{f}\end{array}$ & $\begin{array}{l}\stackrel{2}{\sigma} \\
\stackrel{\infty}{\infty} \\
+\end{array}$ & $\begin{array}{l}8 \\
\stackrel{+}{+} \\
\stackrel{+}{+} \\
+\end{array}$ & $\begin{array}{l}\text { त̂ } \\
\infty \\
\text { ర్ర }\end{array}$ & $\begin{array}{l}\stackrel{n}{2} \\
\stackrel{2}{2} \\
-\end{array}$ & $\begin{array}{l}\stackrel{+}{+} \\
\infty \\
\infty\end{array}$ & $\begin{array}{l}8 \\
\stackrel{1}{*}\end{array}$ & $\begin{array}{l}\stackrel{m}{\tilde{m}} \\
\dot{m}\end{array}$ & $\Xi$ & $\frac{n}{n}$ \\
\hline 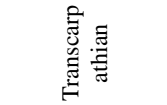 & 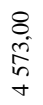 & $\begin{array}{l}\text { fó } \\
\text { চ̊n }\end{array}$ & $\begin{array}{l}0 \\
\vdots \\
8 \\
0 \\
+\end{array}$ & \begin{tabular}{l}
8 \\
8 \\
$\infty$ \\
\multirow{+}{+}{} \\
+
\end{tabular} & 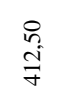 & $\begin{array}{l}8 \\
i n \\
0\end{array}$ & $\begin{array}{l}\infty \\
\stackrel{8}{\Omega}\end{array}$ & के & $\frac{n}{n}$ & o. & 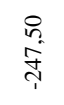 \\
\hline
\end{tabular}


Continuation Table 2

\begin{tabular}{|c|c|c|c|c|c|c|c|c|c|c|c|}
\hline 1 & 2 & 3 & 4 & 5 & 6 & 7 & 8 & 9 & 10 & 11 & 12 \\
\hline Zaporizhia & 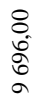 & 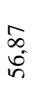 & $\begin{array}{l}\gamma \\
\tilde{\gamma} \\
\partial \\
\infty \\
\infty\end{array}$ & 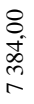 & 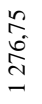 & $\begin{array}{l}8 \\
\text { i } \\
\stackrel{m}{N}\end{array}$ & $\begin{array}{l}\infty \\
\infty \\
\infty \\
\infty \\
\infty\end{array}$ & $\begin{array}{l}0 \\
\text { é }\end{array}$ & $\begin{array}{l}\bar{m} \\
\dot{f}\end{array}$ & $\stackrel{\Xi}{\Xi}$ & 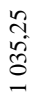 \\
\hline $\begin{array}{l}\text { Ivano- } \\
\text { Frankivsk }\end{array}$ & $\begin{array}{l}8 \\
\infty \\
\dot{f}\end{array}$ & $\begin{array}{l}\stackrel{m}{f} \\
\dot{f}\end{array}$ & $\begin{array}{l}0 \\
n \\
\infty \\
\infty \\
\infty \\
0\end{array}$ & 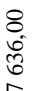 & $\begin{array}{l}\text { n. } \\
\text { हैं } \\
\text { n. }\end{array}$ & $\begin{array}{l}8 \\
\frac{\infty}{7} \\
\frac{1}{10}\end{array}$ & $\begin{array}{l}n \\
\infty \\
i \\
\text { a }\end{array}$ & $\begin{array}{l}\text { dे } \\
\text { ¿े }\end{array}$ & $\begin{array}{l}\text { ה } \\
\text { n. }\end{array}$ & : & 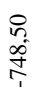 \\
\hline Kyiv & $\begin{array}{l}\text { त̂ } \\
\text { ָี } \\
\infty \\
\infty\end{array}$ & 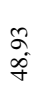 & $\begin{array}{l}n \\
\\
0 \\
\infty \\
\infty \\
0\end{array}$ & $\begin{array}{l}8 \\
+\mathbb{J} \\
\text { J }\end{array}$ & 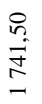 & $\begin{array}{l}\text { r } \\
\infty \\
\infty \\
\sim \\
\sim\end{array}$ & $\begin{array}{l}\infty \\
\infty \\
i\end{array}$ & $\frac{a}{\hat{i}}$ & $\begin{array}{l}\text { ले } \\
\text { है } \\
\text { है }\end{array}$ & $\exists$ & 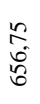 \\
\hline Kirovohrad & 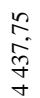 & \begin{tabular}{l} 
ते \\
\multirow{\gamma}{\gamma}{}
\end{tabular} & $\begin{array}{l}n \\
n \\
\infty \\
\infty \\
\infty \\
m\end{array}$ & $\begin{array}{l}8 \\
\text { d. } \\
\text { d. }\end{array}$ & $\begin{array}{l}\text { r. } \\
\text { ș }\end{array}$ & 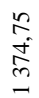 & तై & $\begin{array}{l}\text { Oे. } \\
\text { बें }\end{array}$ & $\begin{array}{l}\tilde{b} \\
\text { iు }\end{array}$ & $\stackrel{\text { an }}{=}$ & $\begin{array}{l}8 \\
\text { no } \\
\frac{1}{2}\end{array}$ \\
\hline Luhansk & $\frac{n}{\frac{n}{n}}$ & 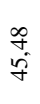 & 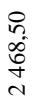 & $\begin{array}{l}8 \\
\text { nू. } \\
\text { n. }\end{array}$ & $\begin{array}{l}8 \\
\text { \&. } \\
0 \\
0\end{array}$ & 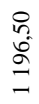 & $\begin{array}{c}m \\
\infty \\
\infty \\
\infty\end{array}$ & $\begin{array}{l}\text { ô } \\
\text { î }\end{array}$ & $\begin{array}{l}\overline{\text { d }} \\
\text { ì }\end{array}$ & 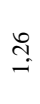 & $\begin{array}{l}\text { n } \\
\text { mi }\end{array}$ \\
\hline Lviv & 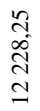 & 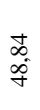 & 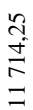 & 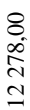 & $\begin{array}{l}8 \\
\dot{+} \\
\text { ti }\end{array}$ & $\begin{array}{l}\stackrel{n}{a} \\
\dot{q}\end{array}$ & $\begin{array}{l}\infty \\
\infty \\
\alpha\end{array}$ & $\begin{array}{l}\vec{f} \\
8 \\
8\end{array}$ & $\begin{array}{l}\text { to } \\
\dot{g}\end{array}$ & $\stackrel{\alpha}{\alpha}$ & 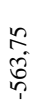 \\
\hline Mykolaiv & $\begin{array}{l}\text { r } \\
\text { î } \\
\stackrel{+}{+}\end{array}$ & $\begin{array}{l}\hat{\infty} \\
\vec{f}\end{array}$ & $\begin{array}{l}n \\
\text { ma } \\
\text { ma } \\
\dot{\gamma}\end{array}$ & $\begin{array}{l}8 \\
\infty \\
\infty \\
\text { ते } \\
m\end{array}$ & $\begin{array}{l}8 \\
b \\
b\end{array}$ & 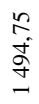 & $\begin{array}{l}\infty \\
0 \\
\infty \\
\infty\end{array}$ & $\begin{array}{l}\text { f } \\
\text { go }\end{array}$ & 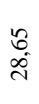 & $\underset{\sim}{\sim}$ & $\begin{array}{l}n \\
\text { c. } \\
\infty \\
\infty\end{array}$ \\
\hline Odesa & 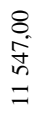 & $\underset{\substack{+ \\
\text { do }}}{ }$ & $\begin{array}{l}\text { â } \\
\text { s. } \\
\text { a } \\
\end{array}$ & $\begin{array}{l}8 \\
- \\
\infty \\
\infty \\
\sigma\end{array}$ & $\begin{array}{l}2 \\
\text { م. } \\
2\end{array}$ & $\begin{array}{l}8 \\
\dot{8} \\
0\end{array}$ & $\begin{array}{l}2 \\
\dot{0} \\
\dot{\infty}\end{array}$ & $\begin{array}{l}5 \\
\text { న } \\
\text { ch }\end{array}$ & $\begin{array}{l}\text { qu } \\
\text { gे }\end{array}$ & $\delta_{-}^{1}$ & $\begin{array}{l}\text { ते } \\
\text { ते }\end{array}$ \\
\hline Poltava & 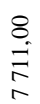 & $\begin{array}{l}n \\
\text { in }\end{array}$ & $\begin{array}{l}\text { in } \\
\text { f } \\
0 \\
0\end{array}$ & $\begin{array}{l}8 \\
\text { तิ } \\
\text { రิ }\end{array}$ & 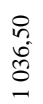 & $\begin{array}{l}8 \\
\stackrel{8}{\Xi} \\
\end{array}$ & $\begin{array}{l}0 \\
h_{0} \\
\infty \\
\infty\end{array}$ & $\underset{-1}{\infty}$ & $\frac{\infty}{v^{\prime}}$ & $\stackrel{-}{-}$ & $\begin{array}{l}\text { rn } \\
\text { i. } \\
\infty \\
m\end{array}$ \\
\hline Rivne & $\begin{array}{l}8 \\
\text { ôे } \\
\text { in } \\
\text { n. }\end{array}$ & $\frac{9}{\dot{q}}$ & $\begin{array}{l}n \\
0 \\
0 \\
0\end{array}$ & $\begin{array}{l}8 \\
\text { in } \\
\text { m } \\
+\end{array}$ & $\begin{array}{l}0 \\
\text { in } \\
\text { sin } \\
\text { in }\end{array}$ & $\begin{array}{l}8 \\
8 \\
\infty \\
\infty\end{array}$ & $\begin{array}{l}a \\
\infty \\
\infty\end{array}$ & D. & $\begin{array}{l} \pm \\
\text { a } \\
\text { m }\end{array}$ & $\stackrel{5}{=}$ & in \\
\hline
\end{tabular}


Continuation Table 2

\begin{tabular}{|c|c|c|c|c|c|c|c|c|c|c|c|}
\hline 1 & 2 & 3 & 4 & 5 & 6 & 7 & 8 & 9 & 10 & 11 & 12 \\
\hline Sumy & $\begin{array}{l}n \\
\text { n. } \\
\infty \\
\text { in }\end{array}$ & $\stackrel{7}{\stackrel{0}{\sigma}}$ & 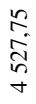 & $\begin{array}{l}8 \\
0 \\
0 \\
0 \\
+\end{array}$ & 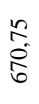 & 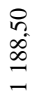 & $\frac{0}{\infty}$ & $\frac{ \pm}{i}$ & $\frac{6}{\sqrt[m]{m}}$ & $\stackrel{9}{=}$ & $\begin{array}{l}\frac{c}{s} \\
\frac{n}{n}\end{array}$ \\
\hline Ternopil & $\begin{array}{l}n \\
n \\
f \\
n \\
n\end{array}$ & $\begin{array}{l}\text { సิ } \\
\text { nิ }\end{array}$ & 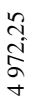 & $\begin{array}{l}8 \\
\frac{8}{n} \\
\frac{1}{n} \\
\end{array}$ & 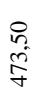 & 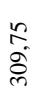 & $\vec{m}$ & $\begin{array}{l}\bar{m} \\
\dot{\alpha}\end{array}$ & $\begin{array}{l}\text { N. } \\
\text { gे }\end{array}$ & s. & $\begin{array}{l}2 \\
\hat{\tilde{\beta}} \\
\frac{1}{1}\end{array}$ \\
\hline Kharkiv & 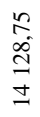 & $\Rightarrow$ & 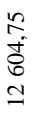 & $\begin{array}{l}\frac{8}{0} \\
= \\
=\end{array}$ & \begin{tabular}{l}
8 \\
8 \\
\multirow{4}{*}{} \\
$=$
\end{tabular} & 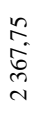 & $\begin{array}{l}\bar{\lambda} \\
\stackrel{\infty}{0}\end{array}$ & $\underset{\infty}{\stackrel{\sim}{\sim}}$ & $\begin{array}{l}\bar{N} \\
\dot{f}\end{array}$ & $\stackrel{5}{s}$ & 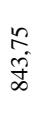 \\
\hline Kherson & $\begin{array}{l}\text { n } \\
\text { î } \\
\text { on } \\
\text { then }\end{array}$ & $\begin{array}{l}\frac{b}{f} \\
\text { s. }\end{array}$ & $\begin{array}{l}\text { ra } \\
\text { o } \\
\infty \\
\infty \\
m\end{array}$ & $\begin{array}{l}8 \\
0 \\
\stackrel{0}{=}\end{array}$ & $\begin{array}{l}\text { a } \\
\text { ț }\end{array}$ & 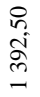 & $\begin{array}{l}\infty \\
\overbrace{0} \\
\infty\end{array}$ & i. & 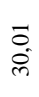 & $\stackrel{\text { I }}{=}$ & $\begin{array}{l}\text { הి } \\
\infty \\
\infty \\
\infty\end{array}$ \\
\hline Khmelnitsky & $\begin{array}{l}\text { D. } \\
\text { ô } \\
\text { in }\end{array}$ & $\begin{array}{l}n \\
m \\
y^{2}\end{array}$ & $\begin{array}{l}n \\
\hat{i} \\
\text { s. } \\
\text { n. }\end{array}$ & $\frac{8}{\frac{\dot{m}}{\sigma}}$ & $\begin{array}{l}n \\
\text { r. } \\
i n \\
\text { in }\end{array}$ & 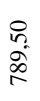 & $\begin{array}{l}\text { ते } \\
\text { \&̊ }\end{array}$ & $\begin{array}{l}\text { స్ } \\
\text { Sీ }\end{array}$ & $\begin{array}{l}\text { aे } \\
\text { iे }\end{array}$ & $\stackrel{2}{0}$ & $\begin{array}{l}\stackrel{n}{\pi} \\
\stackrel{\pi}{\pi}\end{array}$ \\
\hline Cherkasy & $\begin{array}{l}n \\
\text { af } \\
\text { ḋ } \\
n\end{array}$ & $\begin{array}{l}\hat{R} \\
\stackrel{f}{f}\end{array}$ & $\begin{array}{l}\text { n. } \\
\text { \&. } \\
\text { in }\end{array}$ & $\begin{array}{l}8 \\
\stackrel{8}{0} \\
\underset{f}{f}\end{array}$ & $\begin{array}{l}\text { yे } \\
\infty \\
0 \\
0\end{array}$ & 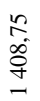 & $\begin{array}{l}\overline{0} \\
\dot{\infty}\end{array}$ & $\begin{array}{l}\check{a} \\
\stackrel{+}{\sim}\end{array}$ & $\begin{array}{l}\text { of } \\
\text { s. } \\
m\end{array}$ & $\stackrel{2}{\beth}$ & $\begin{array}{l}8 \\
\overbrace{}^{\circ} \\
2\end{array}$ \\
\hline Chernivtsi & 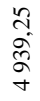 & $\begin{array}{l}\infty \\
\text { iे } \\
\dot{f}\end{array}$ & $\begin{array}{l}2 \\
\hat{2} \\
\hat{\delta} \\
+\end{array}$ & $\begin{array}{l}8 \\
\text { î } \\
\text { in }\end{array}$ & $\begin{array}{l}\text { की } \\
\text { के }\end{array}$ & $\begin{array}{l}n \\
\text { ż } \\
\underline{y}\end{array}$ & $\begin{array}{l}\frac{n}{2} \\
+ \\
\dot{f}\end{array}$ & $\begin{array}{l}\text { aे } \\
\text { si }\end{array}$ & $\begin{array}{l}0 \\
\text { s. } \\
n^{\prime \prime}\end{array}$ & $\delta$ & 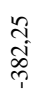 \\
\hline Chernihiv & \begin{tabular}{l}
$n$ \\
2 \\
\multirow{+}{\infty}{} \\
+
\end{tabular} & $\begin{array}{l}\infty \\
\infty \\
o \\
o\end{array}$ & 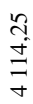 & $\begin{array}{l}8 \\
\text { î } \\
\text { ñ }\end{array}$ & $\begin{array}{l}\text { के } \\
8 \\
8\end{array}$ & $\begin{array}{l}\text { r } \\
\text { di } \\
\underline{m}\end{array}$ & $\begin{array}{l}\text { fo } \\
\text { s. }\end{array}$ & î & $\stackrel{I}{m}$ & $=$ & $\begin{array}{l}\text { â } \\
\text { İ } \\
\text { the }\end{array}$ \\
\hline Kyiv city & $\begin{array}{l}\text { ru } \\
\text { ḋ } \\
0 \\
0\end{array}$ & $\begin{array}{l}\stackrel{8}{\circ} \\
0 \\
0\end{array}$ & $\begin{array}{l}\text { D. } \\
8 \\
\text { N } \\
=\end{array}$ & $\begin{array}{l}0 \\
+ \\
i \\
\text { in }\end{array}$ & 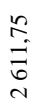 & $\begin{array}{l}\text { तr } \\
0 \\
0 \\
0 \\
\forall\end{array}$ & 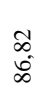 & $\begin{array}{l}\text { مे } \\
\text { gे }\end{array}$ & $\begin{array}{c}\hat{a} \\
\text { nो }\end{array}$ & 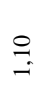 & $\begin{array}{l}0 \\
\text { o. } \\
\text { gे }\end{array}$ \\
\hline
\end{tabular}


The data on the AR Crimea, Sevastopol and temporarily occupied territories of Donetsk and Lugansk regions were not taken into account in the study.

The undertaken analysis of the data given in Table 1 indicates that in terms of regions of the country the indicators of provision of the population with doctors' positions in 2014 had significant differences. Thus, the highest rates of provision by doctors' positions were registered in Kiev (71.19 per 10 thousand people), Zaporizhia (55.61) Kharkiv (54.66), Poltava (53.90), Ivano-Frankivsk (52.83), Chernivtsi (53.48) regions. The lowest rates of provision by doctors' positions were registered in Lugansk (17.02), Donetsk (18.69), Transcarpathian (36.44), Kherson (42.57), Mykolaiv (42.34), Volyn (42.84) regions. The maximum difference reaches 4.18 times. In general, there were registered 194920.5 positions of physicians with an average level of provision 45.59 per 10 thousand people in health care system of Ukraine. In 2014, the health care system of Ukraine employed 159,969.00 physicians with 34951.5 difference between staffing and physical persons of doctors. At the same time, staffing of regular positions taking into account the compatibility in health care system of Ukraine composed $89.39 \%$ with difference from $62.06 \%$ in Lugansk region to $96.76 \%$ in Lviv region. The highest compatibility ratios were registered in Donetsk (1.29), Mykolaiv (1.26) and Kirovohrad, Luhansk, Mykolaiv (1.25) regions. In total, the health care system of Ukraine there are 14262.00 physicians positions staffed on the conditions of compatibility. Most medical positions occupied as part-time are found in Kyiv (2125.75), Dnipropetrovsk (2107.75) and Donetsk (1473.75) regions. Staffing of regular positions by individuals in health care system of Ukraine composed $82.07 \%$ with difference from 49.59\% in Lugansk region to $100 \%$ in Transcarpathian, Lviv, and Chernivtsi regions.

Further, in Table 2 the data on the provision with physicians in 2018 are given.

Analysis of data given in Table 2 indicates that in 2018 in health care system of Ukraine there were registered 191905.25 regular positions of doctors with the level of provision 45.71 per 10 thousand people. In comparison to 2014 the number of regular positions decreased by 3015.25, the level of their availability by 0.12 per 10 thousand people. In terms of regions, the differences in the level of provision with regular doctors' positions by 1.87 times were denoted: from 36.47 in Transcarpathian region to 68.09 in Kyiv.

The gap between the staff posts and individuals of doctors in 2018 reached 35042.25 (in 2014 - 34951.5). The largest shortage of doctors was 
registered in Kyiv (4108.25), Dnipropetrovsk (3817.25), Donetsk (2833.50), Kyiv (2398.25), Kharkiv (2367.75) regions. The surplus of doctors is registered in Ivano-Frankivsk (218), Lviv (50), Chernivtsi (122) oblasts.

The level of regular positions staffing, taking into account the compatibility, reached $87.68 \%$, and the level of staffing by individuals amounted to $81.74 \%$ (in 2014-82.07\%). In terms of administrative territories, the indicated index is substantially different: from $82.07 \%$ in Luhansk to $100 \%$ in Ivano-Frankivsk, Lviv and Chernivtsi regions.

Provision with physical persons of doctors in health care system of Ukraine composes 37.36 per 10 thousand people (in 2014 - 37.41). In terms of administrative territories, the difference is 1.98 times: from 28.21 in Luhansk to 55.72 in Ivano-Frankivsk region.

11398.25 of physicians' posts were occupied on the conditions of compatibility in 2018 (14 262.00 in 2014). The coefficient of compatibility in health care system of Ukraine in 2018 amounted to 1.07.

Next, we analyzed the structure of physicians by the types of medical care. In 2018 the share of physicians of specialized medical care was 79\%, primary medical care $-18 \%$, and emergency medical care $-4 \%$. The analysis conducted allowed to define that the structure of physicians by the types of medical care was almost unchanged over the past 20 years. It should be noted that in the European Union countries the indicated index is 1:3.

It was found in the course of study that in 2018 two-thirds of regular positions of doctors $(65.5 \%)$ belonged to hospitals and only one third $(34.5 \%)$ - to outpatient health care institutions. In EU countries hospitals registered $57 \%$ of the doctors' positions. It was also defined that for the period from 2014 to 2018 the rate of precinct therapists' and precinct pediatricians' positions decrease averaged $12 \%$ and $3 \%$ per year respectively. At the same time, the average annual rate of growth of the number of family physicians is registered at 5\% level. In 2018, the provision of family by doctors amounted to 4.8 per 10 thousand people and in EU countries the indicated index is 7.8 .

Among the most numerous specialties in 2018 (provision of $0,65-2.4$ per 10 thousand population) were obstetricians-gynecologists, hospital therapists, surgeons, anesthesiologists, neurologists, hospital pediatricians, orthopediststraumatologists, roentgenologists, psychiatrists, ophthalmologists and otolaryngologists. The level of provision $0.29-0.64$ per 10 thousand population includes cardiologists, dermatologists, infectious diseases specialists, ultrasound physicians, phthisiatrists, oncologists, neonatologists, urologists, endocrinologist and specialists in functional diagnostics. To doctors with the level of provision $0.1-0.28$ per 10 thousand population belong medicolegal experts, 
physiotherapists, narcologists, pathologists, gastroenterology, endoscopists, rheumatologists (including children's cardio-rheumatologist), pulmonologists, nephrologists, specialists of sports medicine and radiologists. The least numerous specialties (level of provision less than 0.1 per 10 thousand population) include hematologists, transfusiologists, allergists, proctologists, psychotherapists, immunologists, nutritionists, sexologists, toxicologists and sanologues.

For the period from 2014 to 2018 the decrease in the provision by the doctors of the following specialties was registered: urologists, pulmonologists, neurologists, gastroenterologists, surgeons, psychiatrists, rheumatologists, obstetricians, gynecologists, otolaryngologists, transfusiologists, narcologists, dermatologists, ophthalmologists and sexologists, doctors of functional diagnostics, radiologists, psychotherapists, phthisiatrists, toxicologists, immunologists, physiotherapists, specialists of therapeutic sports medicine, hospital paediatricians, hospital therapists, nutritionists and sanologues.

During the same period the increase in the provision of the doctors of the following specialties was registered: cardiologists, nephrologists, endocrinologist, allergists, hematologists, neonatologists, ultrasonic diagnostics doctors, pathologists, infectious diseases specialists, medicolegal experts, anesthesiologists, orthopedists-traumatologists, endoscopy, proctologists and oncologists.

The provision of emergency doctors increased in $2014-2017$ by $0.2 \%$, but in 2018 the decrease of their provision by $1.9 \%$ was registered compared to the previous year rate of 0.98 per 10 thousand population. In 2018 the provision of dentists in Ukraine amounted to 4.0 per 10 thousand population.

For the period 2017-2018 the number of doctors in health care system of Ukraine decreased by 2737 people. Decrease in the number of physicians takes place mainly owing to doctors-stomatologists (45\% of total losses). In 2017-2018 the highest rates of the decrease in the number of doctors was registered in the western boundary regions (Rivne, Volyn, Lviv, IvanoFrankivsk and Transcarpathian), in the south (Mykolaiv and Kherson) and in the east (Dnipropetrovsk and Kharkiv oblasts).

Taking into account that $31 \%$ of population in Ukraine lives in rural areas we have studied the indices of provision of rural population by physicians. It was found that in $20185 \%$ of doctors worked in rural areas. The share of rural physicians ranged from $2 \%$ in Zaporizhia region to $13 \%$ in Transcarpathian and Cherkasy oblasts. The provision of rural population with doctors amounted to 6.6 per 10 thousand of rural population. 
In 2018 the share of pensioners among physicians composed 25\%. Most of the retired doctors work in Kyiv, Cherkasy, Kirovohrad, Mykolaiv, Kherson, Dnipropetrovsk and Donetsk regions.

\section{Provision with mid-level healthcare workers in terms of administrative territories}

At the beginning, we studied and analyzed the data about mid-level health workers by regions for 2014. The obtained data are shown in Table 3.

Analysis of the data given in Table 3 indicates that 360322.75 positions of mid-level medical workers were introduced in 2014 in health care system of Ukraine. Provision of the positions of mid-level medical workers amounted to 84.27 per 10 thousand population. The highest registed indices are registered in the following regions: Kiev (102.69), Sumska (100.77), Chernigov (108.27) region and Kyiv (101.17). The lowest registered indices were registered in the following regions: Lugansk (34.07), Donetsk (38.57) and Transcarpathian (74.25) regions. The maximum index difference was 3.18 times.

339042.5 positions of mid-level medical personnel were occupied in health care system. 21280.25 positions remained not occupied. The system employed 346191.00 mid-level medical workers. The shortage of mid-level health workers amounted to 14131 persons. The largest shortage of midlevel health workers was registered in Kyiv (6 589.25) and Dnipropetrovsk region (3 182.00). At the same time, in the following areas, mid-level medical workers did not work full time: Volyn, Zhytomyr, Transcarpathian, Ivano-Frankivsk, Lviv, Rivne, Khmelnytsky, Chernivtsi regions.

The data on mid-level medical workers for 2018 were studied and analyzed. The data are given in Table 4.

344161.25 positions of secondary medical workers were introduced in health care system of Ukraine in 2018. Level of provision of these posts amounted to 81.98 per 10 thousand population (84.27 in 2014). The difference of the index noted in terms of the regions of the country composed 1.44 times. The highest level of provision with the positions of mid-level medical personnel was registered in Kirovohrad (100.64), Sumy (101.25), Cherkassy (100.43), Chernihiv (104.75) regions. The lowest level of provision with the positions of mid-level medical personnel was registered in Transcarpathian (72.70) and Chernivtsi (80.71) regions. 
Table 3

Mid-level medical workers in the regions of Ukraine, 2014

\begin{tabular}{|c|c|c|c|c|c|c|c|c|}
\hline Region & 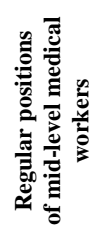 & 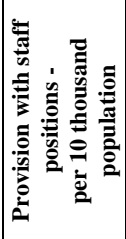 & 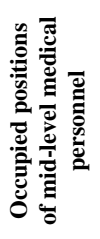 & 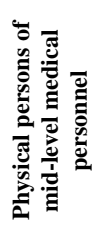 & 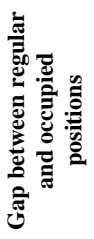 & 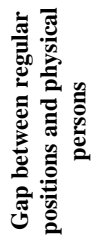 & 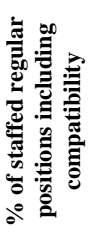 & 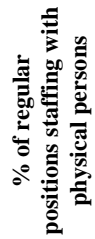 \\
\hline 1 & 2 & 3 & 4 & 5 & 6 & 7 & 8 & 9 \\
\hline Ukraine & $\begin{array}{l}n \\
\text { ñ } \\
\text { ô } \\
\text { हैं }\end{array}$ & $\begin{array}{l}\hat{~} \\
\text { fे }\end{array}$ & $\begin{array}{l}\text { ले } \\
\text { ले } \\
\text { ले }\end{array}$ & $\frac{8}{\frac{8}{2}}$ & 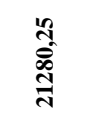 & $\frac{n}{\frac{m}{z}}$ & $\begin{array}{l}\hat{\theta} \\
\dot{\sigma}\end{array}$ & $\begin{array}{l}\stackrel{0}{0} \\
\stackrel{0}{\circ}\end{array}$ \\
\hline Vinnitsa & 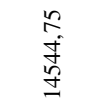 & $\overline{\hat{g}}$ & $\begin{array}{l}n \\
\stackrel{n}{2} \\
\stackrel{2}{=}\end{array}$ & $\begin{array}{l}8 \\
8 \\
2 \\
2\end{array}$ & 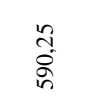 & 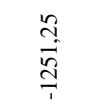 & $\begin{array}{l}\text { बे } \\
\text { ू̆ }\end{array}$ & $\begin{array}{l}0 \\
\stackrel{8}{0}\end{array}$ \\
\hline Volyn & $\begin{array}{l}8 \\
\text { i } \\
\text { on } \\
\sigma\end{array}$ & $\begin{array}{l}\text { ?े } \\
\stackrel{\infty}{\infty}\end{array}$ & $\begin{array}{l}\frac{n}{\sigma} \\
\bar{a}\end{array}$ & $\begin{array}{l}8 \\
\text { - } \\
0 \\
0\end{array}$ & $\begin{array}{l}n \\
8 \\
\Omega\end{array}$ & $\begin{array}{l}8 \\
\text { ते } \\
\stackrel{1}{1}\end{array}$ & 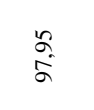 & $\begin{array}{l}\text { ते } \\
\text { J }\end{array}$ \\
\hline Dnipropetrovsk & $\begin{array}{l}8 \\
8 \\
\dot{1} \\
\sim \\
\text { ते }\end{array}$ & $\begin{array}{l}\text { ते } \\
\text { ळ }\end{array}$ & 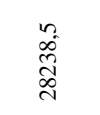 & $\begin{array}{l}8 \\
\text { i } \\
\hat{n} \\
0 \\
0\end{array}$ & $\frac{n}{n}$ & $\begin{array}{l}8 \\
i \\
\infty \\
\infty \\
m\end{array}$ & $\begin{array}{l}n \\
n \\
\kappa\end{array}$ & $\begin{array}{l}\hat{\infty} \\
\stackrel{\infty}{ }\end{array}$ \\
\hline Donetsk & 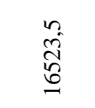 & $\begin{array}{l}n \\
\text { in } \\
\infty \\
\infty\end{array}$ & $\begin{array}{l}8 \\
8 \\
\infty \\
\infty \\
n\end{array}$ & $\begin{array}{l}8 \\
\bar{\Xi} \\
\pm \\
\pm\end{array}$ & $\begin{array}{l}\ddot{2} \\
\stackrel{2}{=}\end{array}$ & $\begin{array}{l}n \\
\text { त̃ } \\
\tilde{N}\end{array}$ & $\vec{n}$ & $\begin{array}{l}\text { qे } \\
\text { هे }\end{array}$ \\
\hline Zhytomyr & 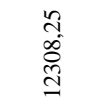 & 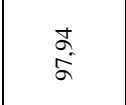 & $\frac{n}{\frac{n}{n}}$ & 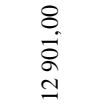 & $\begin{array}{l}n \\
\text { on } \\
m\end{array}$ & $\begin{array}{l}n \\
\text { â } \\
\hat{n}\end{array}$ & $\overrightarrow{\widehat{a}}$ & 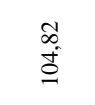 \\
\hline Transcarpathian & $\begin{array}{l}n \\
\text { ñ } \\
\text { ñ }\end{array}$ & \begin{tabular}{l}
$\stackrel{y}{2}$ \\
\multirow{i}{*}{}
\end{tabular} & 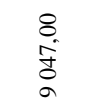 & $\begin{array}{l}8 \\
6 \\
\llcorner \\
0\end{array}$ & $\begin{array}{l}n \\
\tilde{N} \\
\infty\end{array}$ & 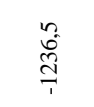 & $\begin{array}{l}\text { مे } \\
\text { ڤ̆ }\end{array}$ & $\begin{array}{l}\stackrel{2}{N} \\
\stackrel{m}{=}\end{array}$ \\
\hline Zaporizhia & 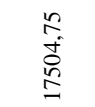 & $\frac{1}{2}$ & $\begin{array}{l}\stackrel{2}{1} \\
\frac{0}{0} \\
0\end{array}$ & $\begin{array}{l}8 \\
\dot{0} \\
0 \\
n\end{array}$ & $\begin{array}{l}n \\
m \\
m \\
m\end{array}$ & $\begin{array}{l}n \\
\stackrel{2}{8} \\
\stackrel{8}{+} \\
+\end{array}$ & $\begin{array}{c}\text { m. } \\
\text { â }\end{array}$ & సે \\
\hline
\end{tabular}


Continuation Table 3

\begin{tabular}{|c|c|c|c|c|c|c|c|c|}
\hline 1 & 2 & 3 & 4 & 5 & 6 & 7 & 8 & 9 \\
\hline $\begin{array}{l}\text { Ivano- } \\
\text { Frankivsk }\end{array}$ & $\begin{array}{l}8 \\
\therefore \\
\infty \\
\infty \\
\simeq\end{array}$ & $\stackrel{\infty}{\sigma^{\prime}}$ & 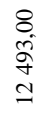 & $\begin{array}{l}8 \\
\text { i } \\
0 \\
\pm\end{array}$ & $\begin{array}{l}8 \\
\text { did }\end{array}$ & $\begin{array}{l}8 \\
8 \\
2 \\
7 \\
7\end{array}$ & $\stackrel{5}{5}$ & 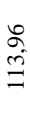 \\
\hline Kyiv & $\begin{array}{l}n \\
\infty \\
0 \\
0 \\
=\end{array}$ & $\begin{array}{l}\text { oे } \\
\text { i }\end{array}$ & 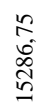 & 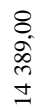 & 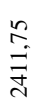 & 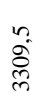 & $\begin{array}{c}\hat{m} \\
\text { - } \\
\infty\end{array}$ & $\frac{m}{\infty}$ \\
\hline Kirovohrad & $\underset{a}{\stackrel{n}{E}}$ & $\stackrel{\text { }}{\stackrel{0}{0}}$ & 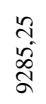 & $\begin{array}{l}8 \\
\stackrel{\leftrightarrow}{\Delta} \\
\text { a }\end{array}$ & $\begin{array}{l}\text { ñ } \\
\text { s. } \\
\text { oे }\end{array}$ & 吕 & $\begin{array}{l}\text { ô. } \\
\text { s. }\end{array}$ & $\begin{array}{l}\infty \\
\dot{\alpha}\end{array}$ \\
\hline Luhansk & $\begin{array}{l}n \\
n \\
\infty \\
\infty \\
i \\
i\end{array}$ & $\begin{array}{l}\hat{0} \\
\dot{m}\end{array}$ & 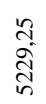 & $\begin{array}{l}8 \\
\stackrel{\infty}{\infty} \\
\frac{\infty}{n}\end{array}$ & $\begin{array}{l}\frac{8}{2} \\
\frac{\vec{m}}{\mathrm{~d}}\end{array}$ & 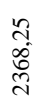 & $\begin{array}{l}\text { å } \\
\text { వై }\end{array}$ & $\begin{array}{l}0 \\
\text { o } \\
0\end{array}$ \\
\hline Lviv & 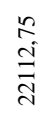 & $\begin{array}{l}\hat{\imath} \\
\stackrel{\infty}{\infty}\end{array}$ & $\begin{array}{l}n \\
\infty \\
\infty \\
\infty \\
\vec{\lambda}\end{array}$ & $\begin{array}{l}8 \\
\stackrel{1}{0} \\
\infty \\
\infty \\
+\end{array}$ & $\begin{array}{l}\text { ते } \\
\text { तิ }\end{array}$ & 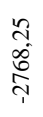 & $\begin{array}{l}\stackrel{0}{\infty} \\
\infty\end{array}$ & în \\
\hline Mykolaiv & $\frac{n}{\stackrel{n}{0}}$ & $\stackrel{1}{\infty}$ & $\begin{array}{l}\frac{n}{2} \\
\frac{j}{a}\end{array}$ & 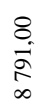 & $\begin{array}{l}8 \\
\text { in } \\
\text { in }\end{array}$ & $\begin{array}{l}n \\
0 \\
\infty \\
\infty\end{array}$ & \begin{tabular}{l}
$n$ \\
\multirow{2}{*}{}
\end{tabular} & हे \\
\hline Odesa & $\begin{array}{l}n \\
\text { ñ } \\
\stackrel{2}{n}\end{array}$ & $\infty$ & $\begin{array}{l}n \\
n \\
\infty \\
\infty \\
\infty\end{array}$ & $\begin{array}{l}8 \\
0 \\
0 \\
0 \\
\infty \\
-\infty\end{array}$ & $\begin{array}{l}8 \\
\text { d } \\
\text { d } \\
\text { N }\end{array}$ & $\begin{array}{l}n \\
\stackrel{n}{\vec{n}}\end{array}$ & $\begin{array}{l}\bar{\lambda} \\
\text { के }\end{array}$ & $\begin{array}{c}\text { oे } \\
\infty \\
\infty\end{array}$ \\
\hline Poltava & $\begin{array}{l}n \\
\hat{a} \\
\hat{\sigma} \\
\end{array}$ & $\hat{a}$ & $\begin{array}{l}\frac{n}{n} \\
\frac{n}{n}\end{array}$ & $\begin{array}{l}8 \\
i \\
\infty \\
m \\
m\end{array}$ & 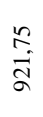 & \begin{tabular}{l} 
an \\
\multirow{2}{*}{}
\end{tabular} & ga & 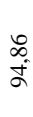 \\
\hline Rivne & 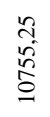 & $\begin{array}{l}\overrightarrow{\hat{j}} \\
\text { S }\end{array}$ & $\begin{array}{l}\text { n } \\
0 \\
0 \\
0 \\
0 \\
0\end{array}$ & $\begin{array}{l}8 \\
8 \\
00 \\
0 \\
0\end{array}$ & 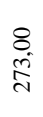 & $\begin{array}{l}n \\
\stackrel{2}{0} \\
\stackrel{1}{1}\end{array}$ & $\begin{array}{l}0 \\
\vdots \\
a \\
a\end{array}$ & : \\
\hline Sumy & 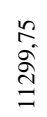 & ह̂. & 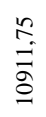 & $\begin{array}{l}8 \\
8 \\
0 \\
= \\
=\end{array}$ & $\begin{array}{l}8 \\
\substack{\infty \\
\infty \\
\infty}\end{array}$ & $\frac{n}{2}$ & $\begin{array}{l}n \\
\text { s. } \\
\sigma^{2}\end{array}$ & $\begin{array}{l}6 \\
8 \\
2\end{array}$ \\
\hline
\end{tabular}


Continuation Table 3

\begin{tabular}{|c|c|c|c|c|c|c|c|c|}
\hline 1 & 2 & 3 & 4 & 5 & 6 & 7 & 8 & 9 \\
\hline Ternopil & $\begin{array}{l}8 \\
\varnothing \\
\& \\
\& \\
0\end{array}$ & $\begin{array}{l}n \\
n \\
0\end{array}$ & $\begin{array}{l}\stackrel{n}{\circ} \\
\stackrel{2}{\circ} \\
\infty\end{array}$ & $\begin{array}{l}8 \\
\frac{8}{5} \\
=\end{array}$ & 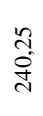 & $\begin{array}{l}8 \\
8 \\
0 \\
0 \\
-1\end{array}$ & $\begin{array}{l}\text { ర్ } \\
\text { \&े }\end{array}$ & $\begin{array}{l}m \\
\stackrel{m}{=}\end{array}$ \\
\hline Kharkiv & $\begin{array}{l}\stackrel{n}{\approx} \\
\tilde{\sigma} \\
\tilde{n}\end{array}$ & $\begin{array}{l}\infty \\
n \\
n \\
\infty\end{array}$ & 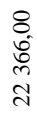 & 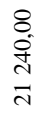 & $\begin{array}{l}\stackrel{n}{n} \\
\stackrel{2}{=}\end{array}$ & $\frac{n}{\stackrel{n}{i}}$ & $\frac{m}{n}$ & mे \\
\hline Kherson & $\frac{n}{\tilde{n}}$ & $\begin{array}{l}\hat{6} \\
\infty \\
\infty\end{array}$ & $\begin{array}{l}n \\
\stackrel{+}{S} \\
\stackrel{5}{2}\end{array}$ & $\begin{array}{l}8 \\
8 \\
\infty \\
\infty \\
\infty \\
\infty\end{array}$ & $\underset{\text { n }}{\stackrel{8}{8}}$ & $\begin{array}{l}n \\
\stackrel{n}{f}\end{array}$ & $\begin{array}{l}\text { o̊ } \\
\text { \&̊ }\end{array}$ & $\stackrel{2}{\approx}$ \\
\hline Khmelnitsky & $\begin{array}{l}\frac{n}{m} \\
=\end{array}$ & $\begin{array}{l}\text { f } \\
\stackrel{\infty}{\infty}\end{array}$ & $\begin{array}{l}8 \\
0 \\
0 \\
0 \\
= \\
=\end{array}$ & $\begin{array}{l}8 \\
0 \\
\hat{n} \\
\end{array}$ & $\begin{array}{l}n \\
\text { ñ } \\
\text { ne }\end{array}$ & $\begin{array}{l}n \\
\text { nू } \\
\text { ñ }\end{array}$ & $\stackrel{3}{5}$ & $\begin{array}{l}\hat{s} \\
\hat{s}\end{array}$ \\
\hline Cherkasy & 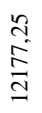 & 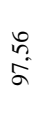 & $\begin{array}{l}8 \\
\text { లె } \\
\infty \\
=\end{array}$ & $\begin{array}{l}8 \\
0 \\
2 \\
\infty \\
=\end{array}$ & $\begin{array}{l}\stackrel{n}{y} \\
\underset{ \pm}{ \pm}\end{array}$ & $\begin{array}{l}2 \\
\text { Iี } \\
\text { In }\end{array}$ & \begin{tabular}{l} 
I \\
\multirow{5}{*}{}
\end{tabular} & $\stackrel{2}{2}$ \\
\hline Chernivtsi & $\begin{array}{l}\stackrel{n}{\circ} \\
\stackrel{\infty}{ \pm} \\
\stackrel{\infty}{\leftarrow}\end{array}$ & $\underset{\infty}{+}$ & \begin{tabular}{l}
$n$ \\
\multirow{2}{N}{} \\
Na
\end{tabular} & $\begin{array}{l}8 \\
\stackrel{8}{2} \\
\infty \\
\infty\end{array}$ & $\begin{array}{l}\text { aे } \\
\text { fे }\end{array}$ & $\begin{array}{l}n \\
n \\
\infty \\
\infty \\
0 \\
+1\end{array}$ & $\frac{2}{\infty}$ & $\begin{array}{l}\text { ô } \\
\stackrel{\Xi}{\beth}\end{array}$ \\
\hline Chernihiv & $\begin{array}{l}\stackrel{n}{0} \\
\stackrel{0}{0} \\
=\end{array}$ & $\begin{array}{l}\widehat{y} \\
\infty \\
0\end{array}$ & $\frac{\mathfrak{n}}{\mathfrak{n}}$ & $\begin{array}{l}8 \\
i n \\
a \\
0\end{array}$ & $\begin{array}{l}n \\
n \\
n \\
n\end{array}$ & $\begin{array}{l}\stackrel{n}{2} \\
\vec{i}\end{array}$ & $\begin{array}{l}\sigma \\
\alpha \\
\dot{\sigma}\end{array}$ & $\begin{array}{l}\text { స̂} \\
\text { ڤ̊ }\end{array}$ \\
\hline Kyiv city & 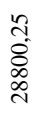 & $\begin{array}{l}= \\
\Xi\end{array}$ & $\frac{n}{2}$ & 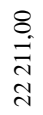 & 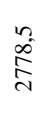 & $\begin{array}{l}2 \\
2 \\
\infty \\
\infty \\
\tilde{b}\end{array}$ & $\begin{array}{l}m \\
\stackrel{m}{2} \\
\overbrace{}^{2}\end{array}$ & $\frac{7}{\approx}$ \\
\hline
\end{tabular}


Table 4

Data on mid-level medical workers in terms of the regions of Ukraine, 2018

\begin{tabular}{|c|c|c|c|c|c|c|c|c|}
\hline Region & 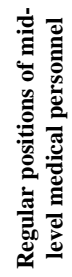 & 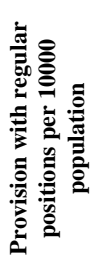 & 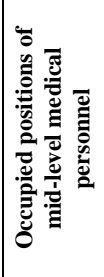 & 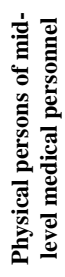 & 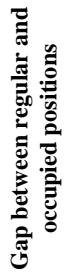 & 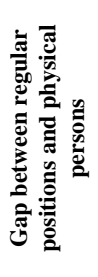 & 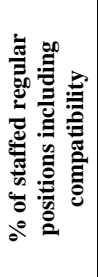 & 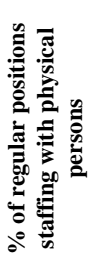 \\
\hline 1 & 2 & 3 & 4 & 5 & 6 & 7 & 8 & 9 \\
\hline Ukraine & 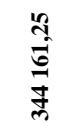 & $\begin{array}{l}\infty \\
\Rightarrow \\
\frac{\infty}{2}\end{array}$ & $\begin{array}{l}\text { in } \\
\text { in } \\
\stackrel{0}{0} \\
\text { co }\end{array}$ & $\begin{array}{l}\stackrel{\Xi}{ } \\
\text { ป̀ } \\
\frac{m}{m}\end{array}$ & 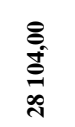 & $\begin{array}{l}\text { A2 } \\
\hat{2} \\
\hat{0}\end{array}$ & \begin{tabular}{l}
$\infty$ \\
\hdashline \\
\hdashline
\end{tabular} & $\begin{array}{l}5 \\
\sigma\end{array}$ \\
\hline Vinnitsia & $\begin{array}{l}n \\
\hat{8} \\
q \\
q \\
=\end{array}$ & $\begin{array}{l}\text { त̂ } \\
\text { ळी }\end{array}$ & $\begin{array}{l}8 \\
\dot{a} \\
\text { I } \\
\simeq\end{array}$ & $\begin{array}{l}8 \\
\overleftarrow{5} \\
0 \\
m \\
\end{array}$ & $\begin{array}{l}\stackrel{n}{2} \\
\stackrel{n}{n}\end{array}$ & 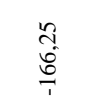 & 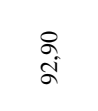 & 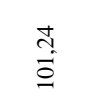 \\
\hline Volyn & 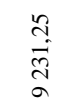 & $\begin{array}{l}q \\
\stackrel{+}{\infty}\end{array}$ & $\begin{array}{l}\stackrel{8}{0} \\
\text { त్ } \\
\infty\end{array}$ & $\begin{array}{l}8 \\
\text { in } \\
\stackrel{1}{5} \\
a\end{array}$ & $\begin{array}{l}\text { ñ } \\
\text { ț }\end{array}$ & 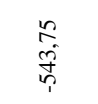 & ra & $\begin{array}{l}\text { oे } \\
\stackrel{0}{0} \\
0\end{array}$ \\
\hline Dnipropetrovsk & $\begin{array}{l}8 \\
\infty \\
\infty \\
\Delta \\
\Delta \\
\Delta\end{array}$ & $\begin{array}{c} \pm \\
\frac{m}{2} \\
\infty\end{array}$ & 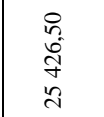 & \begin{tabular}{l}
8 \\
i \\
gे \\
\multirow{2}{7}{}
\end{tabular} & 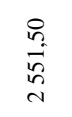 & $\begin{array}{l}8 \\
\text {. } \\
\text { in } \\
+\end{array}$ & $\begin{array}{l}\infty \\
\infty \\
\overbrace{}^{-1}\end{array}$ & $\begin{array}{l}\infty_{0} \\
\infty \\
\infty\end{array}$ \\
\hline Donetsk & 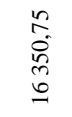 & 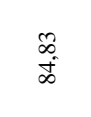 & $\begin{array}{l}n \\
\stackrel{n}{\infty} \\
\stackrel{\infty}{ \pm} \\
\pm\end{array}$ & $\begin{array}{l}8 \\
\stackrel{-}{0} \\
9 \\
=\end{array}$ & 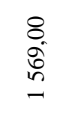 & 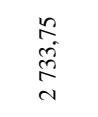 & $\begin{array}{l}q \\
\text { s. }\end{array}$ & 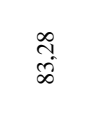 \\
\hline Zhytomyr & $\begin{array}{l}\text { वै } \\
\text { J } \\
\text { J }\end{array}$ & $\begin{array}{l}\vec{\beta} \\
\text { g. }\end{array}$ & $\begin{array}{l}0 \\
\text { ते } \\
\text { o } \\
0\end{array}$ & $\begin{array}{l}8 \\
\stackrel{j}{+} \\
\stackrel{m}{=} \\
=\end{array}$ & $\begin{array}{l}\stackrel{n}{\hat{n}} \\
\stackrel{n}{n}\end{array}$ & $\begin{array}{l}\text { ñ } \\
\alpha^{\infty}\end{array}$ & $\begin{array}{l}\text { f } \\
\text { s. }\end{array}$ & $\frac{ \pm}{\sigma}$ \\
\hline Transcarpathian & $\begin{array}{l}\stackrel{n}{\hat{b}} \\
\stackrel{6}{a} \\
a\end{array}$ & $\begin{array}{l}\stackrel{8}{i} \\
\text { in }\end{array}$ & $\begin{array}{l}8 \\
8 \\
\infty \\
\sigma \\
\infty\end{array}$ & $\begin{array}{l}8 \\
\text { in } \\
\text { a }\end{array}$ & 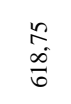 & $\begin{array}{l}\text { aे } \\
\text { ô } \\
\text { ô }\end{array}$ & $\overrightarrow{\widetilde{\sigma}}$ & $\begin{array}{l}\text { fo } \\
\text { fo }\end{array}$ \\
\hline Zaporizhia & 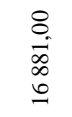 & $\begin{array}{l}\overrightarrow{0} \\
\sigma^{\prime}\end{array}$ & 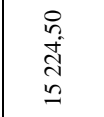 & $\begin{array}{l}8 \\
0 \\
0 \\
0 \\
0\end{array}$ & $\begin{array}{l}0 \\
\text { n. } \\
\text { है } \\
\text { d. }\end{array}$ & $\begin{array}{l}\stackrel{8}{0} \\
\text { i } \\
0 \\
m\end{array}$ & $\frac{2}{8}$ & $\begin{array}{l}0 \\
\underset{\infty}{*}\end{array}$ \\
\hline
\end{tabular}


Continuation Table 4

\begin{tabular}{|c|c|c|c|c|c|c|c|c|}
\hline 1 & 2 & 3 & 4 & 5 & 6 & 7 & 8 & 9 \\
\hline Ivano-Frankivsk & $\begin{array}{l}\stackrel{n}{2} \\
\stackrel{2}{0} \\
\stackrel{m}{\simeq}\end{array}$ & $\begin{array}{l}\stackrel{1}{ } \\
\stackrel{8}{8}\end{array}$ & $\begin{array}{l}8 \\
i \\
2 \\
=\end{array}$ & 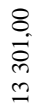 & $\frac{n}{2}$ & 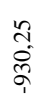 & $\frac{0}{2}$ & $\begin{array}{l}\sqrt{n} \\
\stackrel{0}{0}\end{array}$ \\
\hline Kyiv & 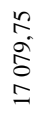 & $\begin{array}{l}\text { مू } \\
\text { ò }\end{array}$ & $\begin{array}{l}\dot{p} \\
m \\
\infty \\
m \\
\dot{D}\end{array}$ & 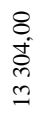 & $\begin{array}{l}\text { a } \\
\text { ó } \\
\text { bे } \\
\text { v }\end{array}$ & $\frac{n}{2}$ & $\begin{array}{l}\overrightarrow{+} \\
\vec{\infty}\end{array}$ & $\begin{array}{l}\text { के } \\
\stackrel{1}{N}\end{array}$ \\
\hline Kirovohrad & 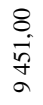 & $\begin{array}{l}\text { ț } \\
8 \\
8\end{array}$ & \begin{tabular}{l}
$n$ \\
\multirow{\delta}{0}{} \\
$\infty$ \\
$\infty$
\end{tabular} & 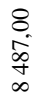 & $\begin{array}{l}\text { a } \\
\dot{f} \\
\text { in }\end{array}$ & $\begin{array}{l}8 \\
\text { dீं }\end{array}$ & $\begin{array}{l} \pm \\
\dot{A}\end{array}$ & $\begin{array}{l}\infty \\
\infty \\
\infty\end{array}$ \\
\hline Luhansk & $\begin{array}{l}\frac{n}{2} \\
\stackrel{2}{2} \\
0\end{array}$ & $\begin{array}{l}\stackrel{2}{1} \\
\infty \\
\infty\end{array}$ & $\begin{array}{l}8 \\
\infty \\
\infty \\
\text { in } \\
\text { in }\end{array}$ & $\begin{array}{l}8 \\
\frac{8}{n} \\
\text { in }\end{array}$ & $\begin{array}{l}n \\
\frac{n}{0} \\
6\end{array}$ & $\frac{n}{2}$ & $\begin{array}{l}\stackrel{2}{2} \\
\stackrel{2}{\infty}\end{array}$ & $\begin{array}{l}n \\
\infty \\
\infty\end{array}$ \\
\hline Lviv & $\begin{array}{l}n \\
\infty \\
\infty \\
\stackrel{+}{+} \\
\vec{N}\end{array}$ & $\underset{\infty}{\infty}$ & 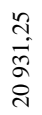 & $\begin{array}{l}8 \\
\text { an } \\
\text { तn } \\
\text { त̃ }\end{array}$ & $\begin{array}{l}\text { in } \\
\text { तn } \\
\text { n. }\end{array}$ & 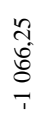 & 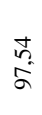 & $\begin{array}{l}\hat{\sigma} \\
\text { d }\end{array}$ \\
\hline Mykolaiv & 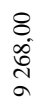 & $\frac{a}{\infty}$ & $\begin{array}{l}\infty \\
\substack{\infty \\
\infty \\
\infty}\end{array}$ & $\underset{i}{\stackrel{8}{i}}$ & $\begin{array}{l}0 \\
\infty \\
\infty \\
\infty \\
\infty\end{array}$ & $\begin{array}{l}8 \\
\stackrel{8}{+} \\
\stackrel{a}{-}\end{array}$ & $\stackrel{f}{\stackrel{8}{8}}$ & $\begin{array}{l}\infty \\
\infty \\
\infty\end{array}$ \\
\hline Odesa & 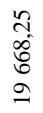 & $\begin{array}{l}\vec{\sigma} \\
\infty_{\infty}\end{array}$ & $\begin{array}{l}n \\
\text { an } \\
\text { vi } \\
\text { I }\end{array}$ & $\begin{array}{l}8 \\
\underset{m}{n} \\
0 \\
0\end{array}$ & $\begin{array}{l}8 \\
0 \\
0 \\
0 \\
1\end{array}$ & $\begin{array}{l}n \\
n \\
m \\
m \\
m\end{array}$ & $\begin{array}{l}R \\
\infty \\
\infty\end{array}$ & $\begin{array}{l}\& \\
+ \\
\infty\end{array}$ \\
\hline Poltava & 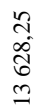 & 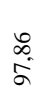 & 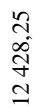 & $\begin{array}{l}8 \\
+ \\
\text { N } \\
\text { I }\end{array}$ & $\begin{array}{l}8 \\
\stackrel{8}{8} \\
\stackrel{-}{8}\end{array}$ & \begin{tabular}{l}
$\tilde{a}$ \\
\multirow{J}{*}{} \\
-
\end{tabular} & $\frac{a}{a}$ & $\frac{ \pm}{8}$ \\
\hline Rivne & $\begin{array}{l}8 \\
-1 \\
\text { f } \\
0\end{array}$ & $\begin{array}{l}\text { ô } \\
\text { a }\end{array}$ & $\begin{array}{l}n \\
\stackrel{n}{-} \\
\stackrel{N}{0} \\
0\end{array}$ & $\begin{array}{l}8 \\
8 \\
\dot{+} \\
=\end{array}$ & $\begin{array}{l}\tilde{2} \\
\hat{\jmath} \\
\dot{\gamma}\end{array}$ & $\begin{array}{l}8 \\
8 \\
0 \\
0 \\
1\end{array}$ & $\begin{array}{l}\infty \\
\infty \\
a\end{array}$ & 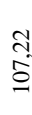 \\
\hline Sumy & 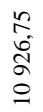 & 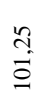 & $\begin{array}{l}8 \\
\stackrel{8}{8} \\
\text { m } \\
0\end{array}$ & $\begin{array}{l}8 \\
\infty \\
\stackrel{+}{+} \\
\stackrel{0}{0}\end{array}$ & $\begin{array}{l}n \\
\hat{\sigma} \\
n \\
n\end{array}$ & $\begin{array}{l}\stackrel{n}{2} \\
\infty \\
o \\
\sigma\end{array}$ & $\begin{array}{l}\stackrel{2}{\circ} \\
\dot{\sigma}\end{array}$ & \begin{tabular}{l}
7 \\
\multirow{2}{\alpha}{}
\end{tabular} \\
\hline Ternopil & $\begin{array}{l}\text { à } \\
\text { à } \\
\text { à }\end{array}$ & $\begin{array}{l}8 \\
\text { a }\end{array}$ & 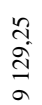 & $\begin{array}{l}8 \\
1 \\
5 \\
0 \\
0\end{array}$ & $\begin{array}{l}8 \\
0 \\
0 \\
0\end{array}$ & $\begin{array}{l}\frac{n}{n} \\
\frac{n}{n}\end{array}$ & $\begin{array}{l}\text { in } \\
\text { nू }\end{array}$ & $\begin{array}{l} \pm \\
\text { है }\end{array}$ \\
\hline
\end{tabular}


Continuation Table 4

\begin{tabular}{|c|c|c|c|c|c|c|c|c|}
\hline 1 & 2 & 3 & 4 & 5 & 6 & 7 & 8 & 9 \\
\hline Kharkiv & 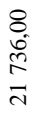 & $\underset{\infty}{\stackrel{N}{+}}$ & 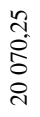 & $\begin{array}{l}8 \\
\text { on } \\
\text { on } \\
0\end{array}$ & $\begin{array}{l}2 \\
2 \\
0 \\
0\end{array}$ & $\begin{array}{l}\frac{8}{\dot{J}} \\
\underset{m}{\vec{j}}\end{array}$ & 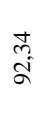 & $\begin{array}{l}n \\
n \\
n \\
\infty\end{array}$ \\
\hline Kherson & 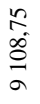 & $\begin{array}{l}8 \\
\frac{8}{\infty}\end{array}$ & $\begin{array}{l}n \\
n \\
\infty \\
\infty \\
\infty \\
\infty\end{array}$ & $\begin{array}{l}8 \\
\text { S. } \\
\text { S- }\end{array}$ & $\begin{array}{l}\text { ते } \\
\text { s. } \\
\text { n. }\end{array}$ & 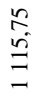 & 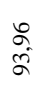 & $\begin{array}{l}n \\
\stackrel{n}{\infty} \\
\infty\end{array}$ \\
\hline Khmelnitsky & $\begin{array}{l}8 \\
\text { o. } \\
= \\
=\end{array}$ & $\begin{array}{l}\stackrel{\Omega}{\tilde{\infty}} \\
\frac{1}{2}\end{array}$ & $\begin{array}{l}\text { r } \\
\text { s. } \\
\vdots \\
0\end{array}$ & 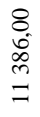 & $\begin{array}{l}n \\
n \\
n\end{array}$ & $\begin{array}{l}8 \\
\text { } \\
\text { ते }\end{array}$ & $\begin{array}{l}n \\
m \\
s\end{array}$ & $\begin{array}{l}\text { ț } \\
\text { di }\end{array}$ \\
\hline Cherkasy & 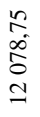 & $\begin{array}{l}\text { qu } \\
8 \\
8\end{array}$ & 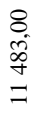 & $\begin{array}{l}8 \\
+ \\
\infty \\
\infty \\
\varrho\end{array}$ & $\begin{array}{l}n \\
\text { s. } \\
\text { on }\end{array}$ & $\begin{array}{l}\text { 足 } \\
+\underset{d}{+}\end{array}$ & $\begin{array}{l}5 \\
\dot{a} \\
\text { a }\end{array}$ & $\begin{array}{l}\hat{2} \\
\infty \\
\infty\end{array}$ \\
\hline Chernivtsi & \begin{tabular}{l}
$n$ \\
\multirow{f}{c}{} \\
d \\
$r$
\end{tabular} & $\stackrel{\overrightarrow{0}}{\vec{\infty}}$ & 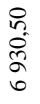 & $\begin{array}{l}8 \\
\infty \\
\infty \\
\infty \\
\infty\end{array}$ & $\begin{array}{l}\text { ? } \\
\text { f } \\
\text { f }\end{array}$ & $\begin{array}{l}\text { तે } \\
\text { o } \\
0 \\
1\end{array}$ & $\begin{array}{l}\text { ते } \\
\text { घे }\end{array}$ & $\begin{array}{l}= \\
\equiv\end{array}$ \\
\hline Chernihiv & $\begin{array}{l}8 \\
8 \\
0 \\
+1 \\
0\end{array}$ & 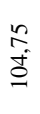 & $\begin{array}{l}\text { ra } \\
\text { f } \\
\infty \\
\sigma\end{array}$ & $\begin{array}{l}8 \\
8 \\
\infty \\
0 \\
0\end{array}$ & $\begin{array}{l}\stackrel{2}{a} \\
\overline{0}\end{array}$ & $\begin{array}{l}8 \\
\text { i. } \\
n \\
n\end{array}$ & $\begin{array}{l}\mathbb{I} \\
\text { J }\end{array}$ & $\begin{array}{c}\text { ले } \\
\text { से }\end{array}$ \\
\hline Kyiv city & $\begin{array}{l}2 \\
\hat{\sigma} \\
\vec{b} \\
\vdots\end{array}$ & $\begin{array}{l}\tilde{z} \\
\dot{\alpha}\end{array}$ & $\begin{array}{l}n \\
\text { ñ } \\
\text { co } \\
\text { ते }\end{array}$ & $\begin{array}{l}8 \\
\text { i } \\
\text { ते } \\
\text { in }\end{array}$ & $\begin{array}{l}8 \\
+ \\
\text { d. } \\
\text { m. }\end{array}$ & $\begin{array}{l}\stackrel{n}{r} \\
\text { ñ } \\
\stackrel{n}{r}\end{array}$ & $\begin{array}{l}4 \\
\infty \\
\infty\end{array}$ & 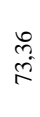 \\
\hline
\end{tabular}

In total, 315057 positions of secondary health workers are occupied with 313412.00 physical persons.

The level of staffing of the regular positions of medical workers taking into account the compatibility reached $91.83 \%$, and $91.07 \%$ by physical persons. The lowest level of staffing of the regular positions by individuals is registered in Kyiv (73.36\%) and Kyiv (77.89\%) region.

In the following regions of Ukraine definite part of mid-level medical workers are not employed full time: Vinnytsia, Volyn, Transcarpathian, Ivano-Frankivsk, Lviv, Rivne, Ternopil, Khmelnytsky, Chernivtsi.

In 2018 the shortage of secondary health workers in health care system of Ukraine amounted to 30749 individuals (14131 in 2014). The highest shortage of secondary medical personnel was registered in health care institutions of Kyiv (7 357), Dnipropetrovsk (4 526), Kyiv (3 775), Odesa (3 135), Kharkiv (3 141) and Zaporizhia (3 012) regions. 
Next the issue on mid-level medical personnel working in rural areas was studied. The obtained data are shown in Table 5.

13295 doctor's assistant and obstetrical centers functioned in Ukraine in 2018 for providing the rural population with pre-medical medical care. 537 doctor's assistant and obstetrical centers of their total number are not staffed with any medical professional. The largest number of not staffed doctor's assistant and obstetrical centers is registered in Chernihiv (73), Poltava (59), Dnipropetrovsk (50) and Sumy (44) regions.

All doctor's assistant and obstetrical centers in Ivano-Frankivsk and Chernivtsi regions are staffed with medical personnel.

Table 5

Data on mid-level medical personnel working in rural areas in terms of the regions of Ukraine, 2018

\begin{tabular}{|c|c|c|c|}
\hline Region & $\begin{array}{c}\text { Number of doctor's } \\
\text { assistant and } \\
\text { obstetrical center }\end{array}$ & $\begin{array}{l}\text { Number of doctor's } \\
\text { assistant and obstetrical } \\
\text { centers without mid- } \\
\text { level medical personnel }\end{array}$ & $\begin{array}{c}\text { Number of } \\
\text { mid-level } \\
\text { medical } \\
\text { personnel in } \\
\text { rural areas }\end{array}$ \\
\hline Ukraine & 13295,00 & 537,00 & 43273,00 \\
\hline Vinnitsia & 881,00 & 24,00 & 2817,00 \\
\hline Volyn & 764,00 & 22,00 & 1873,00 \\
\hline Dnipropetrovsk & 402,00 & 50,00 & 1878,00 \\
\hline Donetsk & 327,00 & 35,00 & 479,00 \\
\hline Zhitomir & 816,00 & 11,00 & 2332,00 \\
\hline Transcarpathian & 280,00 & 9,00 & 2763,00 \\
\hline Zaporizhia & 359,00 & 13,00 & 1100,00 \\
\hline Ivano-Frankivsk & 531,00 & - & 2040,00 \\
\hline Kyiv & 643,00 & 7,00 & 1852,00 \\
\hline Kirovohrad & 483,00 & 15,00 & 1288,00 \\
\hline Luhansk & 231,00 & 19,00 & 536,00 \\
\hline Lviv & 1004,00 & 1,00 & 3064,00 \\
\hline Mykolaiv & 385,00 & 33,00 & 1141,00 \\
\hline Odesa & 505,00 & 29,00 & 2204,00 \\
\hline Poltava & 624,00 & 59,00 & 1805,00 \\
\hline Rivne & 623,00 & 4,00 & 1849,00 \\
\hline Sumy & 519,00 & 44,00 & 1465,00 \\
\hline Ternopil & 733,00 & 7,00 & 2044,00 \\
\hline Kharkiv & 499,00 & 40,00 & 1767,00 \\
\hline Kherson & 348,00 & 9,00 & 1248,00 \\
\hline Khmelnitsky & 909,00 & 23,00 & 2933,00 \\
\hline Cherkasy & 540,00 & 10,00 & 2179,00 \\
\hline Chernivtsi & 212,00 & - & 1403,00 \\
\hline Chernihiv & 677,00 & 73,00 & 1213,00 \\
\hline
\end{tabular}


43273.00 mid-level medical workers were employed in rural areas in 2018. The largest number of mid-level medical professionals worked in rural areas in the following regions: Lviv (3064.00), Khmelnytsky (2933.00), Vinniytsia (2817.00), Transcarpathian (2763.00). The smallest number of mid-level medical professionals worked in rural areas in the following regions: Donetsk (479.00) and Lugansk (536.00).

It was determined in the course of study hat the ratio of the number of nurses and doctors in Ukraine tends to decrease with the index 1:2.0. In the countries of the European Union the ratio of doctors and nurses composes 1:4.1.

The number of mid-level medical personnel decreased by 17032 persons for the period 2017-2018. It is more than twice as much as in the previous year. At the same time, the number of obstetricians decreased by $8.4 \%$, the number of doctors' assistants decreased by $5.4 \%$, and nurses - by $4.3 \%$.

It was found in the course of the study that the share of medical nurses, who work at the primary medical care level, grows in the structure of secondary medical personnel. In 2014-2018 staffing with nurses increased almost by 1.5 times (from 4.7 to 6.5 per10 thousand population). This influenced the ratio of the number of nurses and physicians in health care institutions of primary level: in 2014 one family doctor accounted for 1.2 nurses, and in $2018-1.4$ nurses.

In 2018 the share of retirement age persons among mid-level medical personnel amounted to $13 \%$. The highest proportion of retirement age persons among mid-level medical personnel are observed in Kyiv, Cherkasy, Kirovohrad, Mykolayiv, Kherson, Dnipropetrovsk, and Donetsk regions. At the same time, in 2015-2018 the share of abiturients in the specialty "Nursing" of the level of junior specialist, which includes programs for training nurses, doctors' assistants and midwives, has been decreasing every year: from 3.70 to 2.64 per 10 thousand population. In 2018 the one abiturient in specialty "Nursing" accounted for 3.6 working pensioners among the mid-level medical personnel.

\section{CONCLUSIONS}

The article is focused on basic problems of personnel provision in health care system of Ukraine. The dynamics for 2014-2018 years of population provision in terms of the regions of Ukraine with physicians and mid-level medical workers are presented. Provision of rural population with physicians and mid-level medical workers is specially presented. The deficiency of physicians and mid-level medical personnel is shown in terms of regions. The author shows that the largest outflow of medical personnel from health 
care system of Ukraine was registered for the years 2017-2018. Taking into consideration that the personnel potential is the most significant component of the resource provision of health care system, the national health care system faces the task of optimizing human policies in health care system of the country.

\section{SUMMARY}

The undertaken analysis of personnel resources in health care system of Ukraine shows that in 2018 there were registered 191905.25 of regular positions of doctors with the level of provision 45.71 per 10 thousand population. In comparison to 2014 the number of regular positions decreased by 3015.25 , the level of their availability by 0.12 per 10 thousand people. In terms of regions, the differences in the level of provision with regular doctors' positions by 1.87 times were denoted: from 36.47 in Transcarpathian region to 68.09 in Kyiv. The gap between the regular posts and individuals of doctors in 2018 reached 35042.25 (34951.5 in 2014).

The level of regular positions staffing, taking into account the compatibility, reached $87.68 \%$, and the level of staffing by individuals amounted to $81.74 \%$ (82.07\% in 2014). 11398.25 of physicians' posts were occupied on the conditions of compatibility in 2018 (14 262.00 in 2014). The index of compatibility in health care system of Ukraine in 2018 amounted to 1.07 .

It was found in the course of study that in 2018 two-thirds of regular doctors' positions $(65.5 \%)$ belonged to hospitals and only one third (34.5\%) - to outpatient health care institutions. In EU countries 57\% of the doctors' positions were registered in hospitals. For the period 2017-2018 the number of doctors in health care system of Ukraine decreased by 2737 people, in 2018 in rural areas $5 \%$ of doctors worked.

344161.25 positions of secondary medical workers were introduced in health care system of Ukraine in 2018. Level of provision of these posts amounted to 81.98 per 10 thousand population (84.27 in 2014). The difference of the index noted in terms of the regions of the country composed 1.44 times.

In total, there are 316057 occupied positions of secondary health workers with 313412.00 physical persons. The shortage of secondary health workers in health care system of Ukraine in 2018 amounted to 30749 individuals (14131 in 2014). It was determined in the course of study that the ratio of the number of nurses and physicians in Ukraine tends to decrease with the index 1:2.0. 
The geographical distribution in the provision of physicians and nurses is uneven. The low availability of both physicians and nurses is registered in Transcarpathian, Kiev, Mykolaiv, Kherson, Donetsk and Luhansk regions.

It should be noted that in statistical statements used to plan personnel policies and human resources management, only a minimal set of indicators is used of the total number gathered by medical institutions. There are no aspects of staff turnover, their retention and satisfaction, efficiency and lifelong learning. Disaggregation is limited by gender and age, as well as in terms of urban/rural areas. There is no unified electronic register of medical personnel. National statistical reports on medical personnel are not harmonized with international standards. All that complicates the comparison of Ukrainian data as for human resources of health care system with other countries.

\section{REFERENCES}

1. Junious D.L. et al. (2004) A study of school nurse job satisfaction. Journal of School Nursing, no. 20, pp. 88-93.

2. Sovmestnaya rabota na blago zdorov'ya. Doklad o sostoyanii zdravookhraneniya v mire (2006) [Work together for Health. World Health Report]. Retrieved from: https://www.who.int/topics/health_workforce/ru/

3. Razvitie medicinskih kadrov [Medical personnel development]. WHO: website. Retrieved from: http://whodc.mednet.ru/ru/component/attachments/ download/20.html (accessed 13 November 2019).

4. Gupta N. et al. (2003) Uses of population census data for monitoring geographical imbalance in the health workforce: snapshots from three developing countries. International Journal for Equity in Health, no. 2, p. 11.

5. Maier, C. B., \& Aiken, L. H. (2016) Task shifting from physicians to nurses in primary care in 39 countries: a cross-country comparative study. European Journal of Public Health, no. 26 (6), pp. 927-934.

6. Boerma W.G.W., Kringos D.S., Wiegers T.A., Baltag V., \& Khimion, L. (2010) Evaluation of structure and provision of primary care in Ukraine: a survey-based project in the regions of Kiev and Vinnitsa.

7. Ghalijenko L. I. (2011) Suchasni kadrovi problemy okhorony zdorov'ja $v$ krajinakh Jevropejsjkogho reghionu [Modern problems of health care personnel in the countries of European Region] WHO. Skhidnojevropejsjkyj zhurnal ghromadsjkogho zdorov'ja, no. 1 (13), pp. 279-281.

8. Kadry zdravoohraneniya 2030. Globalnaya strategiya dlya razvitiya kadrovyh resursov zdravoohraneniya [Health personnel 2030. Global strategy for health workforce development] WHO website. Retrieved from: 
https://www.who.int/hrh/resources/russian_global_strategyHRH.pdf (accessed 12 November 2019).

9. Forcier MB et al. (2004). Impact, regulation and health policy implications of physician migration in OECD countries. Human Resources for Health, 2:12.

10. Diallo K. Data on the migration of health-care workers: sources, uses, and challenges (2004) Bulletin of the World Health Organization, no. 82, pp. 559-636.

11. Commonwealth Code of Practice for the International Recruitment of Health Workers (2003) London, Commonwealth Secretariat. Retrieved from: http://www.thecommonwealth.org/shared_asp_files/uploadedfiles/ \{7BDD970B-53AE-441D-81DB-1B64C37E992A\}_CommonwealthCodeof Practice.pdf, (accessed 15 April 2008).

12. Rasshirenie dostupa $\mathrm{k}$ rabotnikam zdravoohraneniya $\mathrm{v}$ otdalennyh $\mathrm{i}$ selskih rajonah. Rekomendacii po globalnoj politike. [Increased access to health workers in remote and rural areas. Global policy recommendations]. WHO website. Retrieved from: http://www.who.int/entity/hrh/retention/ retention_recommendations_ru.pdf (accessed 11 November 2019).

13. Ocenka finansirovaniya, obrazovaniya, upravleniya i politicheskogo konteksta dlya strategicheskogo planirovaniya kadrovyh resursov zdravoohraneniya [Assessment of financing, education, governance and policy context for strategic health workforce planning]. WHO website. Retrieved from: http://apps.who.int/gb/ebwha/pdf_files/WHA59REC1/r/Part2-Res-ru.pdf) (accessed 14 November 2019).

14. Lekhan V.M., Krjachkova L.V. (2017) Naukove obgruntuvannja suchasnykh pidkhodiv do pidghotovky kerivnykh medychnykh kadriv [Scientific substantiation of modern approaches to training of medical officials and executives] Proceedings of the Kadrova polityka u sferi okhorony zdorov'ja $\mathrm{v}$ umovakh zaghroz nacionaljnij bezpeci Ukrajiny (Ukraine, Kiev, March 23, 2017), Kiev, pp. 86-9.

15. Slabkyj Gh.O. (2018) Kadrova polityka [Personnel policy] Retrieved from: http://www.uiph.kiev.ua/index.asp?p=information $\& s=2$ (accessed 14 November 2019).

16. Vezhnovecj T.A. (2014) Do pytannja vyznachennja kryterijiv profesijnogho psykhologhichnogho doboru kerivnykiv zakladiv okhorony zdorov'ja [Issues on determination of the criteria for professional psychological choice of health care managers] Ukrajinsjkyj zhurnal $z$ problem medycyny praci, no 1, pp. 28-36.

17. Voronenko V., Feshhenko I. (2011) [Personnel is a key to success] Vashe zdorov'ja, no 49. Retrieved from: http:// www.vz.kiev.ua/?p=3813. 
18. Vasjuk N.O. (2015) Udoskonalennja kadrovogho zabezpechennja derzhavnogho upravlinnja okhoronoju zdorov ja v Ukrajini [Improvement of staffing of state health care management in Ukraine] Zbirnyk naukovykh pracj Nacionaljnoji akademiji derzhavnogho upravlinnja pry Prezydentovi Ukrajiny Vol. 2, pp. 34-51.

19. Nacionaljna strateghija reformuvannja systemy okhorony zdorov'ja $v$ Ukrajini na period 2015-2020 rokiv [National Health Care Reform Strategy in Ukraine for 2015-2020] Retrieved from: https://moz.gov.ua/ uploads/0/691-strategiya.pdf.

20. Pro skhvalennja Koncepciji reformy finansuvannja systemy okhorony zdorov'ja, Rozporjadzhennja Kabinetu Ministriv Ukrajiny №1013-r vid 30.11.2016 [On the approval of the Concept of Healthcare Financing Reform, Order of the Cabinet of Ministers of Ukraine № 1013-p of 30.11.2016] Retrieved from: https://zakon.rada.gov.ua/laws/show/10132016-p.

21. Derzhavnyj zaklad «Centr medychnoji statystyky Ministerstva okhorony zdorov'ja Ukrajiny [State Institution «Center for Medical Statistics of the Ministry of Health of Ukraine»]. Retrieved from: http://medstat.gov.ua.

22. Boghdan D., Bojko A., Vasyljkova A. ta in. (2019) Kadrovi resursy systemy okhorony zdorov'ja v Ukrajini. Sytuacijnyj analiz [Human resources of the health care system in Ukraine. Situational analysis] Proekt USAID «Pidtrymka reformy okhorony zdorov’ja» Kyjiv, 133 p.

\section{Information about the author:} Slabkiy G. O., orcid.org/0000-0003-2308-7869 Professor at the Department of Health Sciences

Uzhhorod National University 29, Mytna str., 88000, Uzhhorod, Ukraine 